\title{
High-Temperature Switchable Nonlinear Optical and Dielectric Material Revealed by Molecular Modification
}

Yongfa Xie, Jingjing Zheng, Kuangqi Zheng, Qing Liu, Jie Duan, Wei Chen, Shuke Shao, Hu Cai*

College of Chemistry, Nanchang University, Nanchang 330031, China.

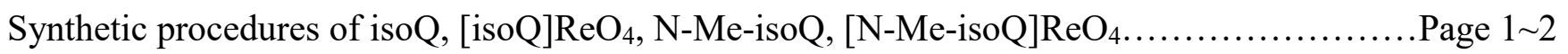

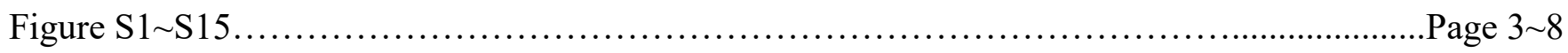

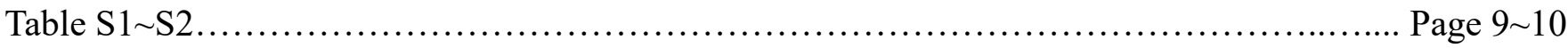

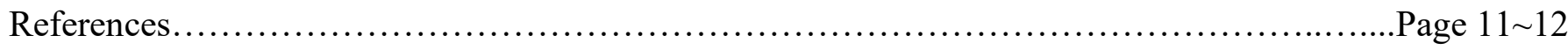

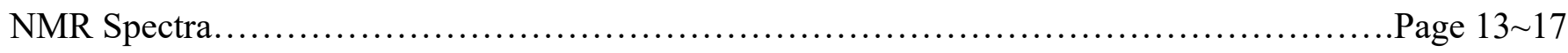

\section{Synthetic procedures:}

\section{Synthesis of 2-azabicyclo [2.2.2] octane(isoquinuclidine)}

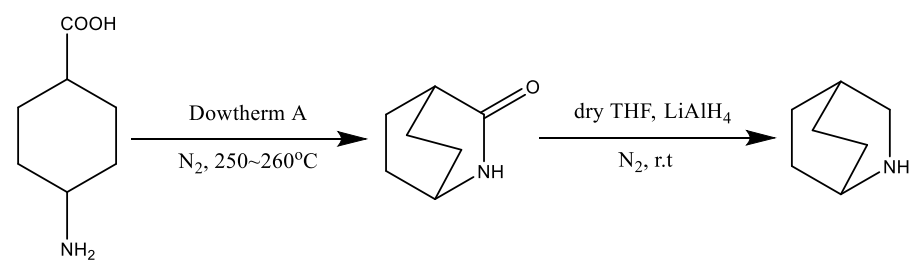

Scheme S1. Synthetic route of isoquinuclidine

Synthesis of 3-isoquinuclidone: A solution of 4-aminocyclohexene carboxylic acid (7.531 g, $50 \mathrm{mmol})$ in Dowtherm A $(150 \mathrm{~mL})$ was heated to $250 \sim 260^{\circ} \mathrm{C}$ and refluxed for 1 hour. The generated water was removed by a Dean Stark. Then the reaction mixture was cooled to room temperature and adjust the $\mathrm{pH}$ value to $4 \sim 5$ with hydrochloric acid. The solution is extracted with water $(50 \mathrm{~mL} * 3)$. The $\mathrm{pH}$ value of the combined water phases was adjusted to $10 \sim 11$ with $\mathrm{NaOH}$ and then extracted with $200 \mathrm{~mL} \mathrm{CH}_{2} \mathrm{Cl}_{2}(200 \mathrm{~mL} * 3)$. The combined organic phases were dried over $\mathrm{Na}_{2} \mathrm{SO}_{4}$, filtered, and concentrated to afford a gray white solid powder $(2.9 \mathrm{~g}$, yield: 46\%) (without further purification for next step). ${ }^{1} \mathrm{H}$ NMR $\left(300 \mathrm{MHz}, \mathrm{D}_{2} \mathrm{O}\right) \delta 3.76-3.72(\mathrm{~m}, 1 \mathrm{H}), 2.53$ $2.49(\mathrm{~m}, 2 \mathrm{H}), 1.87-1.64(\mathrm{~m}, 8 \mathrm{H}) .{ }^{13} \mathrm{C} \mathrm{NMR}\left(75 \mathrm{MHz}, \mathrm{D}_{2} \mathrm{O}\right) \delta 181.73,47.49,36.94,26.32,23.19$.

Preparation of [3-O-isoQ] $\mathrm{ReO}_{4}$ : 3 -isoquinuclidone ( $5 \mathrm{mmol}$ ) was completely dissolved in water, and then perrhenic acid ( $5 \mathrm{mmol}$ ) was dropped into the mixture. The mixture was filtered, and the fitration was left standing at room temperature for one week to obtain colorless transparent crystals, but their crystallinity is not enough for single-crystal X-ray diffraction. ${ }^{1} \mathrm{H}$ NMR $\left(300 \mathrm{MHz}, \mathrm{D}_{2} \mathrm{O}\right) \delta 3.74-3.70(\mathrm{~m}, 1 \mathrm{H}), 2.52-2.48(\mathrm{~m}, 2 \mathrm{H})$, 1.84-1.64 (m, 8H). ${ }^{13} \mathrm{C}$ NMR (75 MHz, $\left.\mathrm{D}_{2} \mathrm{O}\right) \delta 181.74,47.55,36.81,26.28,23.18$. 
Synthesis of isoquinuclidine: 3 -isoquinuclidone $(0.625 \mathrm{~g}, 5.0 \mathrm{mmol})$ was added to anhydrous THF (15 $\mathrm{mL})$ under nitrogen atmosphere. Then $\mathrm{LiAlH}_{4}(1 \mathrm{M}$ in THF, $5.5 \mathrm{~mL})$ was gradually added into the solution under stirring at room temperature, and the reaction was stirred for 10 hours. At the end of the reaction, water was added gradually to quench the reaction and then filtered through a pad of celite to remove the pasty aluminum salts and washed with ethyl acetate (EA) $(30 \mathrm{~mL})$. Water phase of the obtained filtration was removed by separating funnel. Then $\mathrm{HCl}(1 \mathrm{M}$ in ethyl acetate, $6 \mathrm{~mL})$ was added into the organic phase, and the mixture was concentrated in vacuum. The obtained residue solid was sublimated to obtain a light yellow solid, 2-azabicyclo[2.2.2] octan-2-ium chloride (0.427g, yield: 58\%). ${ }^{1} \mathrm{H}$ NMR (400 MHz, $\left.\mathrm{D}_{2} \mathrm{O}\right) \delta 3.49-3.45$ $(\mathrm{m}, 1 \mathrm{H}), 3.25-3.24(\mathrm{~m}, 2 \mathrm{H}), 2.02-1.73(\mathrm{~m}, 9 \mathrm{H}) .{ }^{13} \mathrm{C} \mathrm{NMR}\left(101 \mathrm{MHz}, \mathrm{D}_{2} \mathrm{O}\right) \delta$ 47.64, 47.43, 24.51, 24.35, 24.30.

Crystal preparation of [isoQ] $\mathrm{ReO}_{4}$ : Isoquinuclidine $(5 \mathrm{mmol})$ was dissolved in a mixed solution of methanol/ water $(2 / 1)$, then perrhenic acid $(5 \mathrm{mmol})$ was added. The mixture was stirred evenly, and then filtered. Slow evaporation of the filtration gave the colorless transparent bulk crystals at room temperature within a week. Initial melting point, $329.0{ }^{\circ} \mathrm{C} .{ }^{1} \mathrm{H}$ NMR (400 MHz, $\left.\mathrm{D}_{2} \mathrm{O}\right) \delta 3.48-3.45(\mathrm{~m}, 1 \mathrm{H}), 3.25-3.24$ $(\mathrm{m}, 2 \mathrm{H}), 2.11-1.70(\mathrm{~m}, 9 \mathrm{H}) .{ }^{13} \mathrm{C}$ NMR $\left(101 \mathrm{MHz}, \mathrm{D}_{2} \mathrm{O}\right) \delta 45.11,44.90,21.97,21.82,21.76$.

\section{Synthesis of 2-methyl-2-azabicyclo[2.2.2]octane}

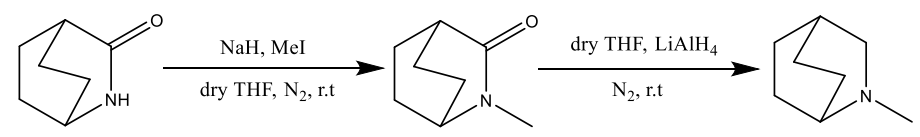

Scheme S2. Synthetic route of 2-methyl-2-azabicyclo[2.2.2]octane

Synthesis of 2-methyl-2-azabicyclo[2.2.2] octan-3-one: $\mathrm{NaH}(1.20 \mathrm{~g}, 40 \mathrm{mmol})$ was added to a solution of 3-isoquinuclidone $(2.502 \mathrm{~g}, 20 \mathrm{mmol})$ in anhydrous THF $(100 \mathrm{~mL})$ under nitrogen atmosphere. The mixture was refluxed and stirred for 2 hours under nitrogen atmosphere. The reaction mixture was cooled to room temperature, then MeI $(2.5 \mathrm{~mL}, 40 \mathrm{mmol})$ was added, and the reaction was stirred for three hours at room temperature. After the reaction completed, water $(50 \mathrm{~mL})$ was added to quench the reaction, and the solution was extracted with $\mathrm{CHCl}_{3}(100 \mathrm{~mL} * 3)$. The combined organic phases were dried over $\mathrm{Na}_{2} \mathrm{SO}_{4}$, filtered, concentrated and purified by flash chromatography on silica gel using EA:MeOH=10:1 as the eluent to afford a light yellow solid (2.140 g, yield: 77\%). ${ }^{1} \mathrm{H}$ NMR (400 MHz, $\left.\mathrm{D}_{2} \mathrm{O}\right) \delta 3.75-3.72(\mathrm{~m}, 1 \mathrm{H}), 3.02(\mathrm{~s}, 3 \mathrm{H}), 2.59$ $2.56(\mathrm{~m}, 1 \mathrm{H}), 1.86-1.68(\mathrm{~m}, 8 \mathrm{H}) .{ }^{13} \mathrm{C} \mathrm{NMR}\left(101 \mathrm{MHz}, \mathrm{D}_{2} \mathrm{O}\right) \delta 179.12,56.06,37.47,31.58,25.37,23.58$.

Synthesis of 2-methyl-2-azabicyclo[2.2.2] octane: At room temperature, $\mathrm{LiAlH}_{4}(1 \mathrm{M}$ in THF, $16.5 \mathrm{~mL})$ was added dropwise to the stirring solution of 2-methyl-2-azabicyclo[2.2.2] octan-3-one (2.086 g, $15 \mathrm{mmol})$ in anhydrous THF $(50 \mathrm{~mL})$ under nitrogen, and the reaction was stirred for 5 hours. After the reaction completed, water was added gradually to quench the reaction, then the reaction mixture was filtered through a pad of celite to remove the pasty aluminum salts and washed with EA $(50 \mathrm{~mL})$. Water phase of the obtained filtration was removed by separating funnel. Then $\mathrm{HCl}(1 \mathrm{M}$ in EA, $16 \mathrm{~mL})$ was added into the organic phase, and the mixture was concentrated in vacuum. The obtained residue solid was sublimated to obtain a white solid, 2-methyl-2-azabicyclo[2.2.2] octan-2-ium chloride (1.308g, 54\%). ${ }^{1} \mathrm{H}$ NMR $\left(400 \mathrm{MHz}, \mathrm{D}_{2} \mathrm{O}\right) \delta 3.60$ $3.54(\mathrm{~m}, 1 \mathrm{H}), 3.34-3.30(\mathrm{~m}, 1 \mathrm{H}), 2.93(\mathrm{dt}, J=12 \mathrm{~Hz}, 2 \mathrm{~Hz}, 1 \mathrm{H}), 2.87(\mathrm{~s}, 3 \mathrm{H}), 2.17-1.64(\mathrm{~m}, 9 \mathrm{H}) .{ }^{13} \mathrm{C}$ NMR $(101$ $\left.\mathrm{MHz}, \mathrm{D}_{2} \mathrm{O}\right) \delta 56.33,53.84,40.51,23.22,23.19,21.91,21.04,17.79$.

Crystal preparation of [N-Me-isoQ] $\mathrm{ReO}_{4}$ : 2-methyl-2-azabicyclo[2.2.2] octane (5mmol) was completely dissolved in water, and then perrhenic acid $(5 \mathrm{mmol})$ was dropped into the mixture. The mixture was filtered, and the filtration was left standing at room temperature for one week to obtain colorless transparent crystals. Initial melting point, $221.0{ }^{\circ} \mathrm{C} .{ }^{1} \mathrm{H}$ NMR $\left(400 \mathrm{MHz}, \mathrm{D}_{2} \mathrm{O}\right) \delta 3.63-3.56(\mathrm{~m}, 1 \mathrm{H}), 3.36-3.31(\mathrm{~m}, 1 \mathrm{H}), 2.95(\mathrm{dt}$, $J=12 \mathrm{~Hz}, 2 \mathrm{~Hz}, 1 \mathrm{H}), 2.89$ (s, 3H), 2.21-1.65 (m, 9H). ${ }^{13} \mathrm{C}$ NMR $\left(101 \mathrm{MHz}, \mathrm{D}_{2} \mathrm{O}\right) \delta 56.38,53.84,40.52,23.27$, $23.23,21.95,21.07,17.81$. 


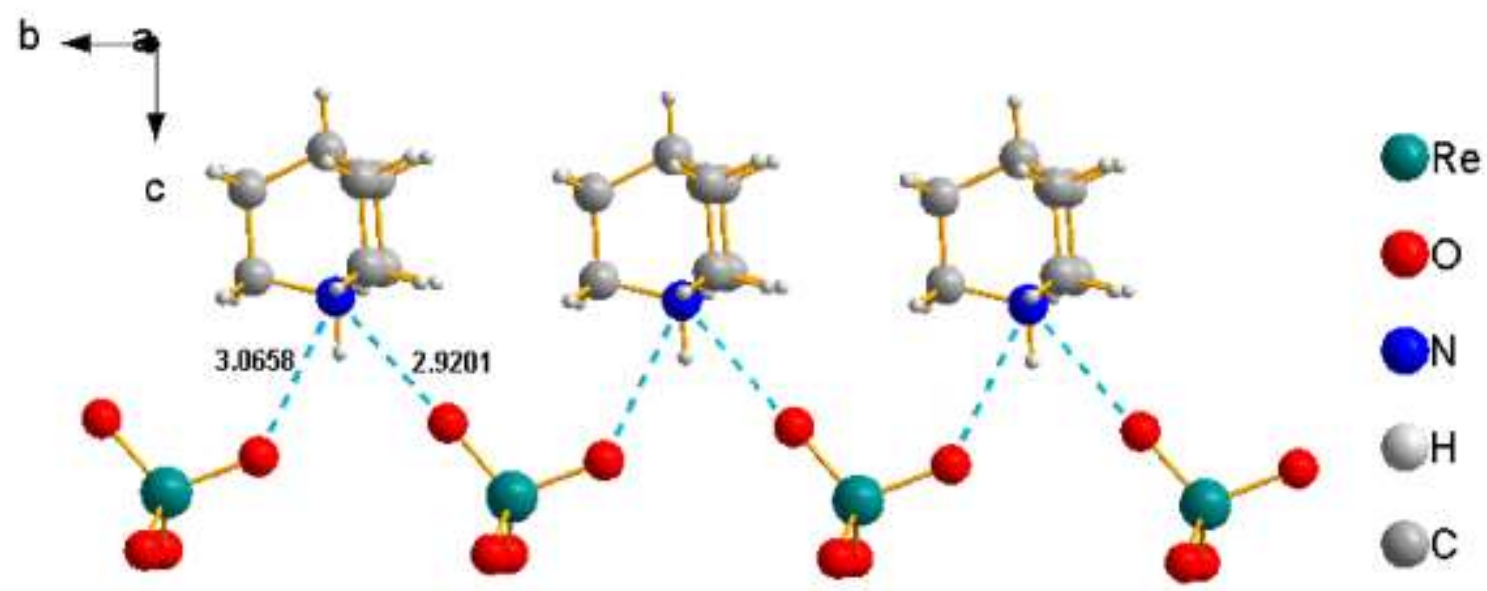

Figure S1. The distances of bifurcated hydrogen bonds in $\mathrm{HQReO}_{4}$ crystal are $2.9201 \AA$ and $3.0658 \AA$.

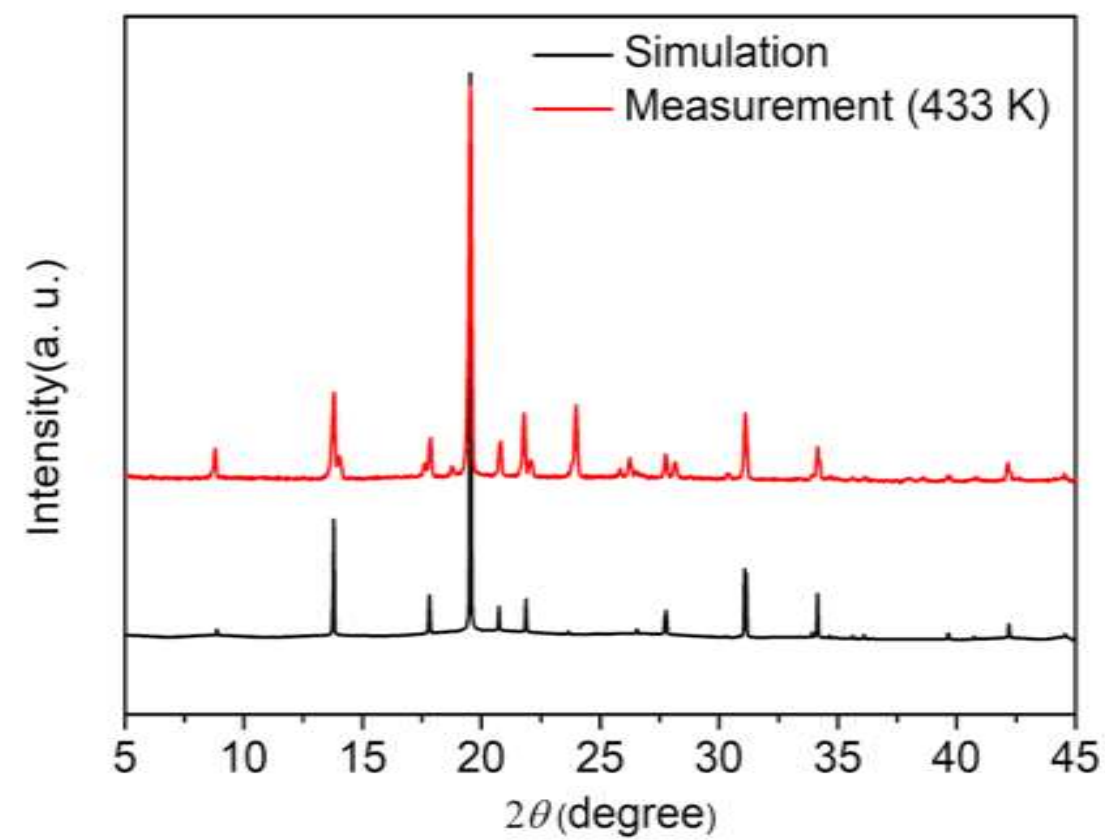

Figure S2. The measured PXRD (red) at $433 \mathrm{~K}$ and simulated PXRD of [N-Me-isoQ]ReO 4 by Materials Studio software.

(a)

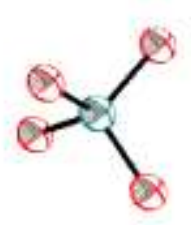

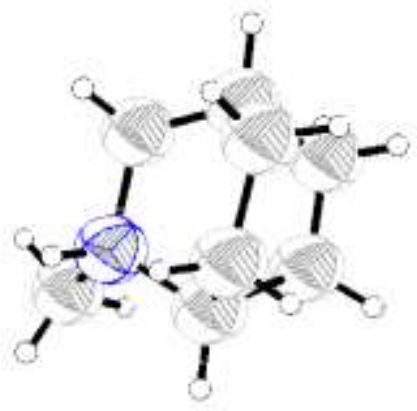

(b)
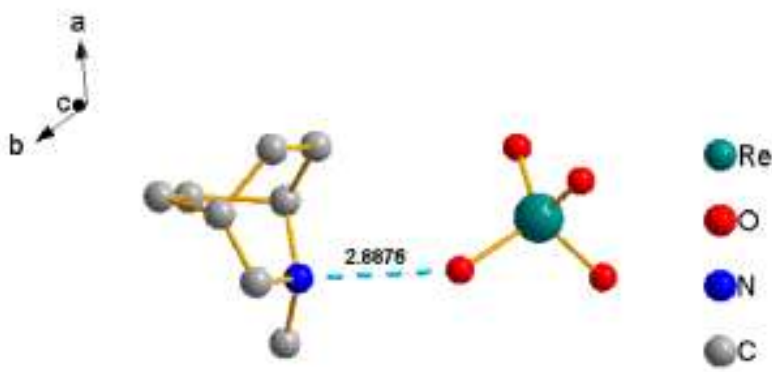

Figure S3. (a) Crystal structures (at $300 \mathrm{~K}$ ) of $[\mathrm{N}-\mathrm{Me}-\mathrm{isoQH}] \mathrm{ReO}_{4}$ in the asymmetric unit with the displacement ellipsoids drawn. (b) The distance of one hydrogen bond in $[\mathrm{N}-\mathrm{Me}-\mathrm{isoQH}] \mathrm{ReO}_{4}$ is $2.8876 \AA$. 
(a)

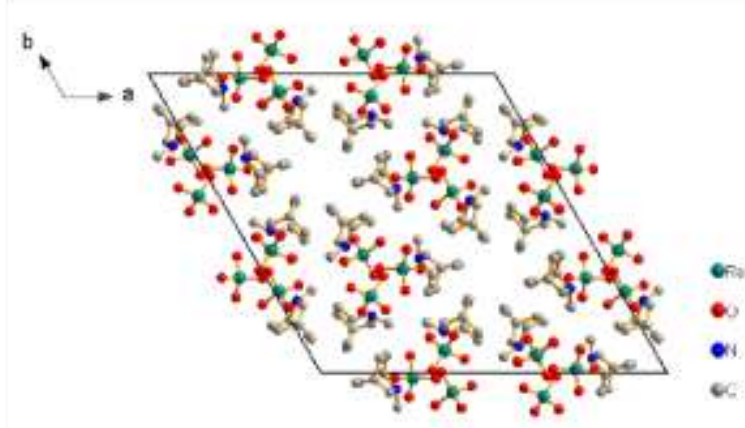

(b)

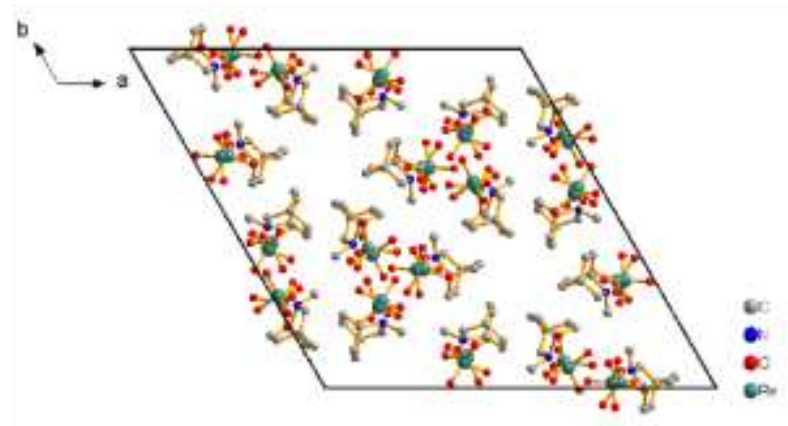

Figure S4. Crystal packing diagram of $[\mathrm{N}-\mathrm{Me}-\mathrm{isoQH}] \mathrm{ReO}_{4}$ from $c$-axis at $300 \mathrm{~K}$ (a) and $333 \mathrm{~K}$ (b).

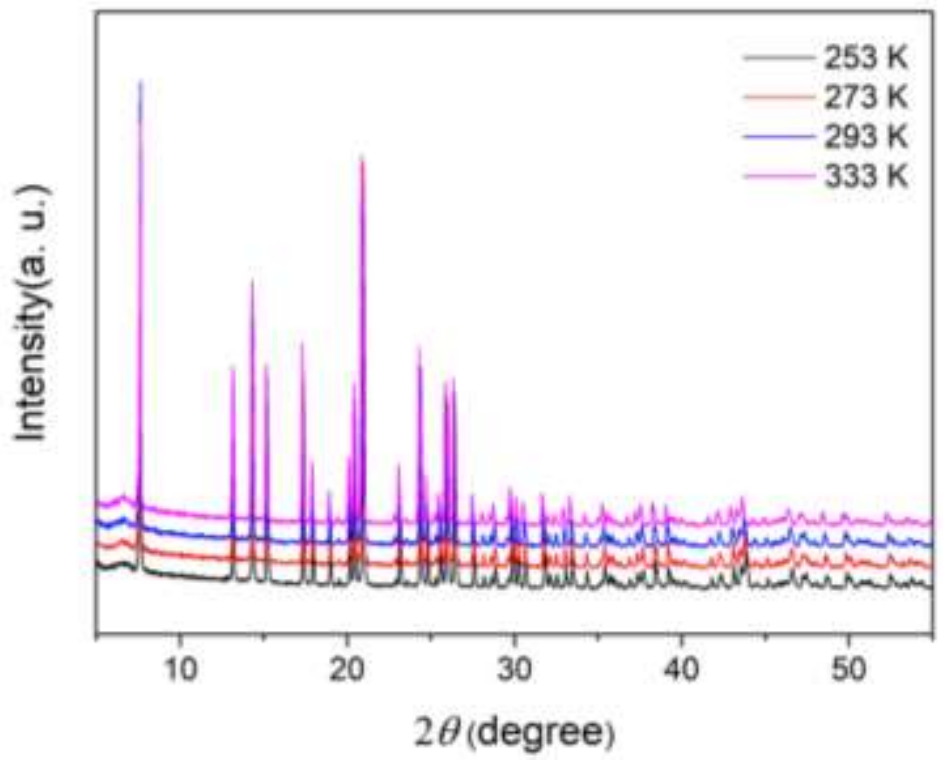

Figure S5. Variable-temperature powder X-ray diffraction (PXRD) patterns of $[\mathrm{N}-\mathrm{Me}-\mathrm{isoQH}] \mathrm{ReO}_{4}$.

(a)

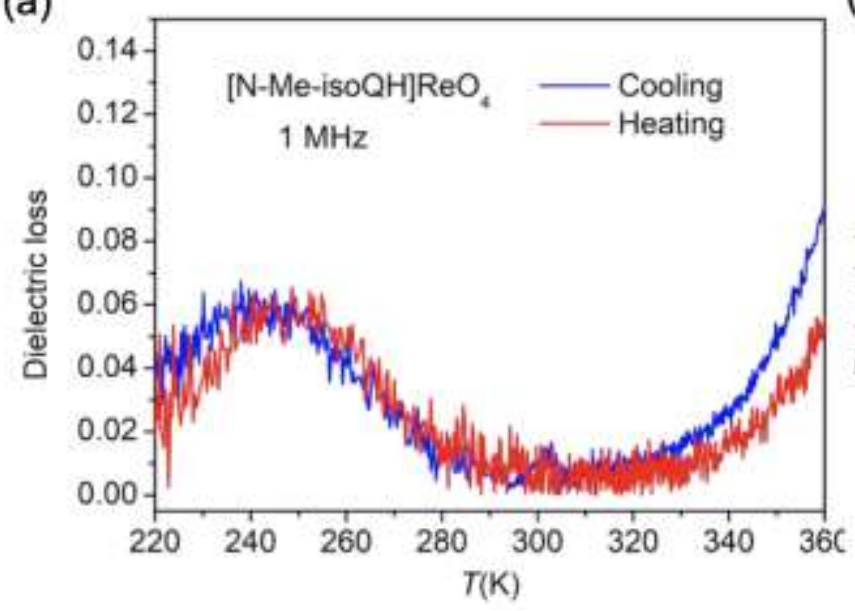

(b)

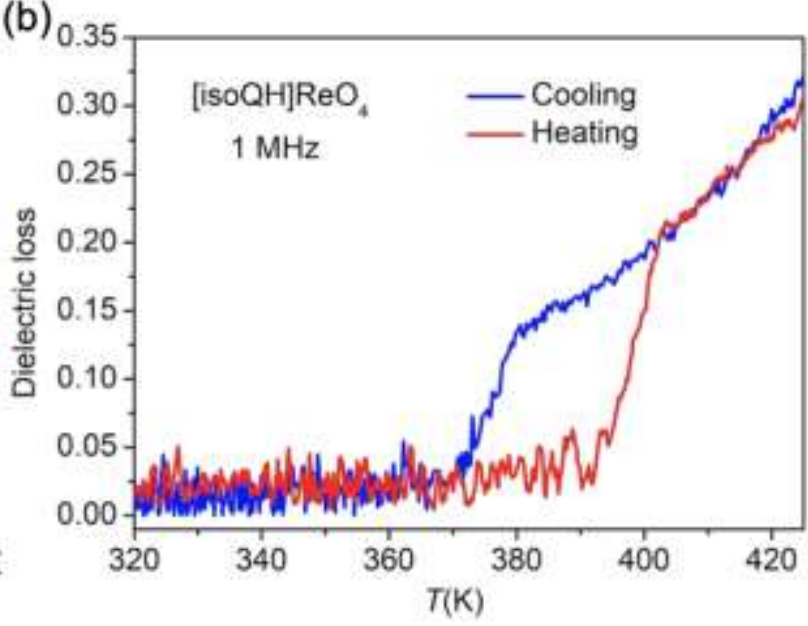

Figure S6. Temperature dependences of the dielectric losses of [N-Me-isoQH] $\mathrm{ReO}_{4}$ and $[\mathrm{isoQH}] \mathrm{ReO}_{4}$. 


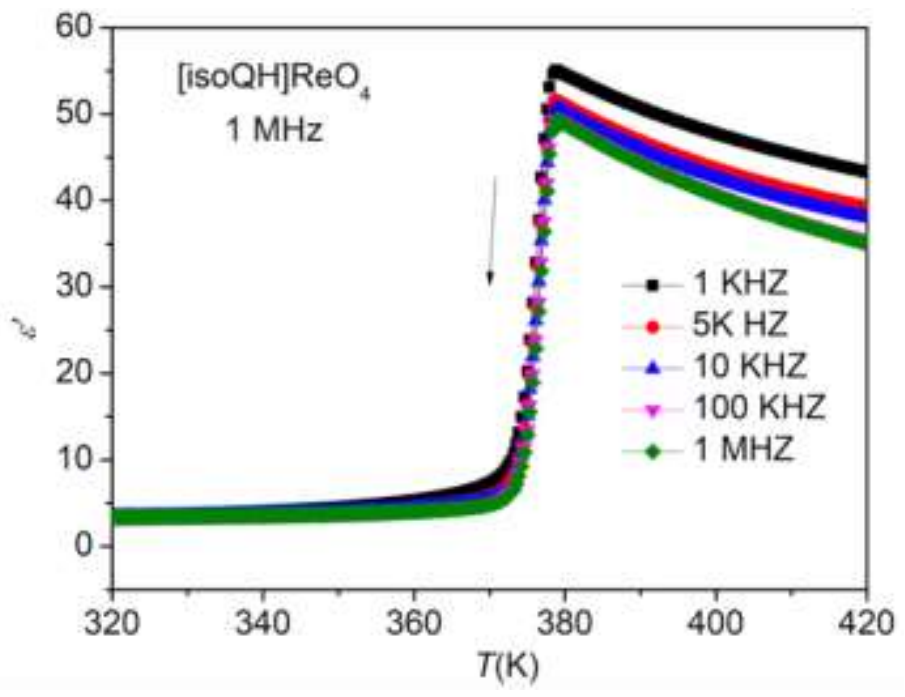

Figure S7. The dielectric measurement of $[\mathrm{isoQH}] \mathrm{ReO}_{4}$ in the cooling run at different frequencies.

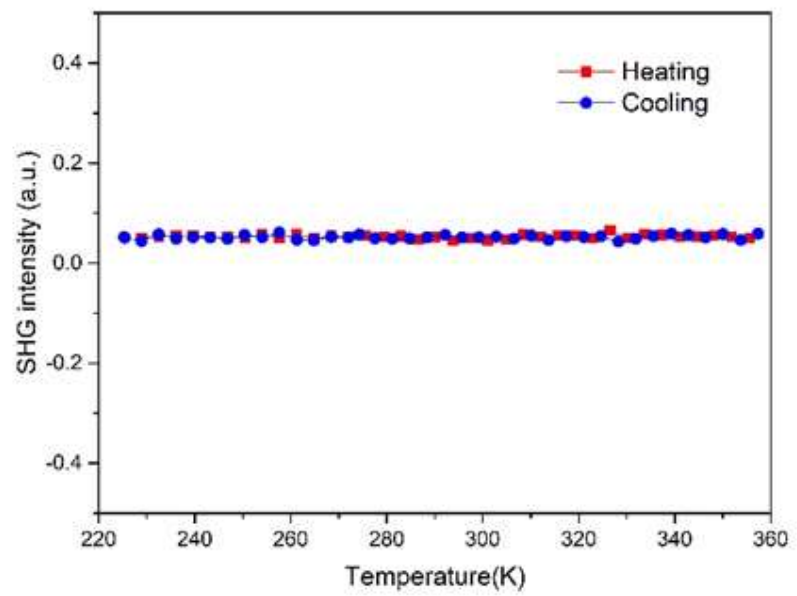

Figure S8. Temperature dependent SHG intensity of [N-Me-isoQH]ReO4.

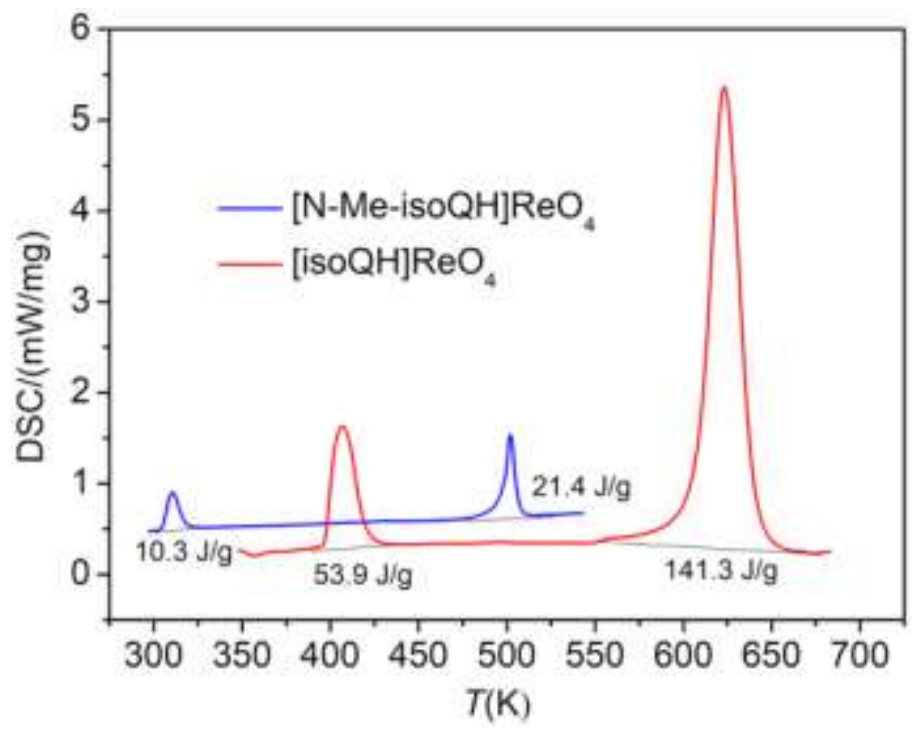

Figure S9. The DSC measurement over the melting points of [N-Me-isoQH] $\mathrm{ReO}_{4}$ (blue line) and [isoQH] $\mathrm{ReO}_{4}$ (red line). 
(a)

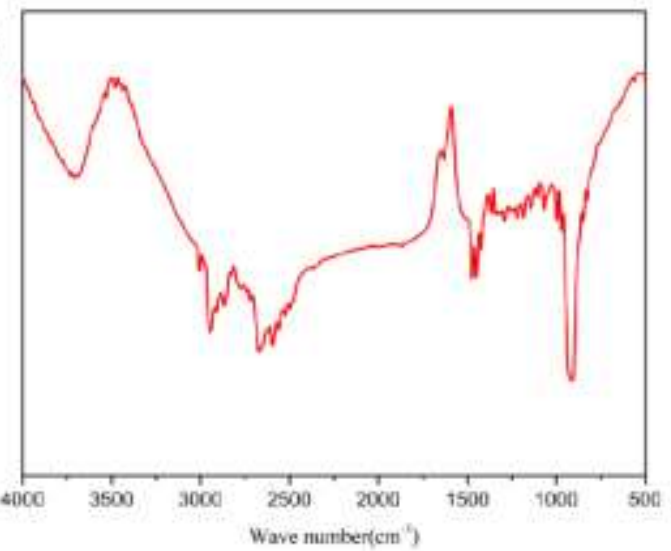

(b)

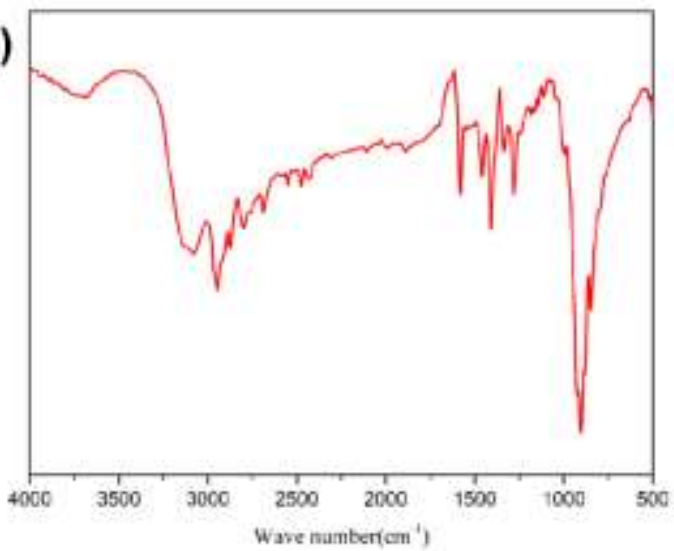

Figure S10. The IR spectrums for compound $[\mathrm{N}-\mathrm{Me}-\mathrm{isoQH}] \mathrm{ReO}_{4}(\mathrm{a})$ and $[\mathrm{isoQH}] \mathrm{ReO}_{4}(\mathrm{~b})$.

(a)

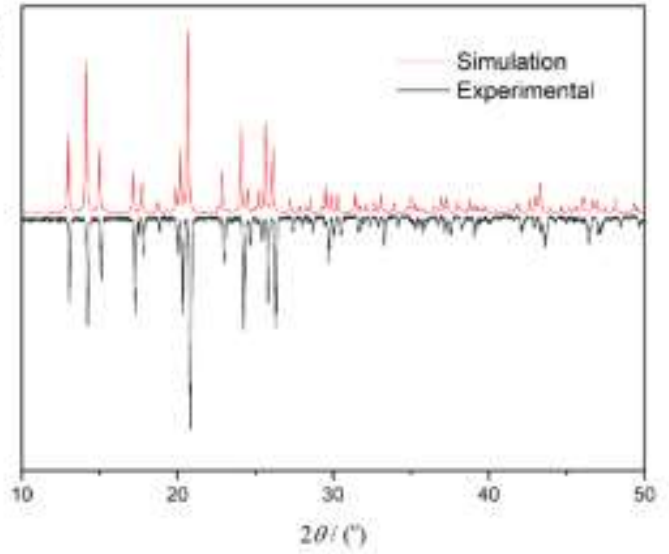

(b)

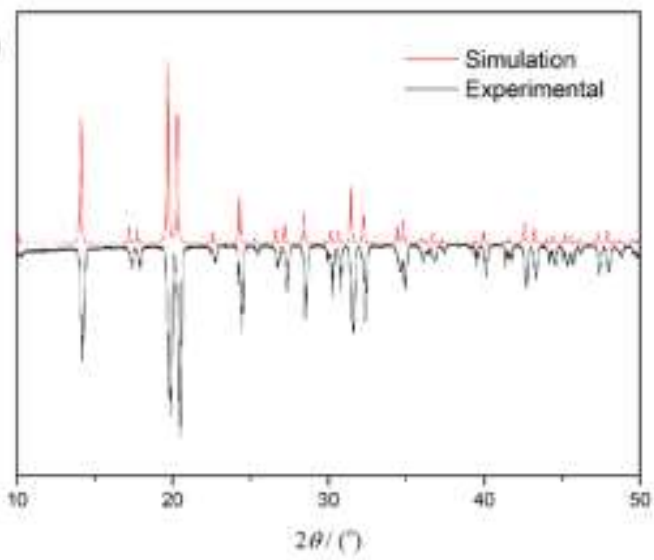

Figure S11. The powder X-ray diffraction (PXRD) patterns of [N-Me-isoQH] ReO 4 (a) and $\left[\right.$ isoQH] $\mathrm{ReO}_{4}(\mathrm{~b})$ closely match the simulated ones respectively, thereby verifying the phase purity of their bulk phases.

(a)

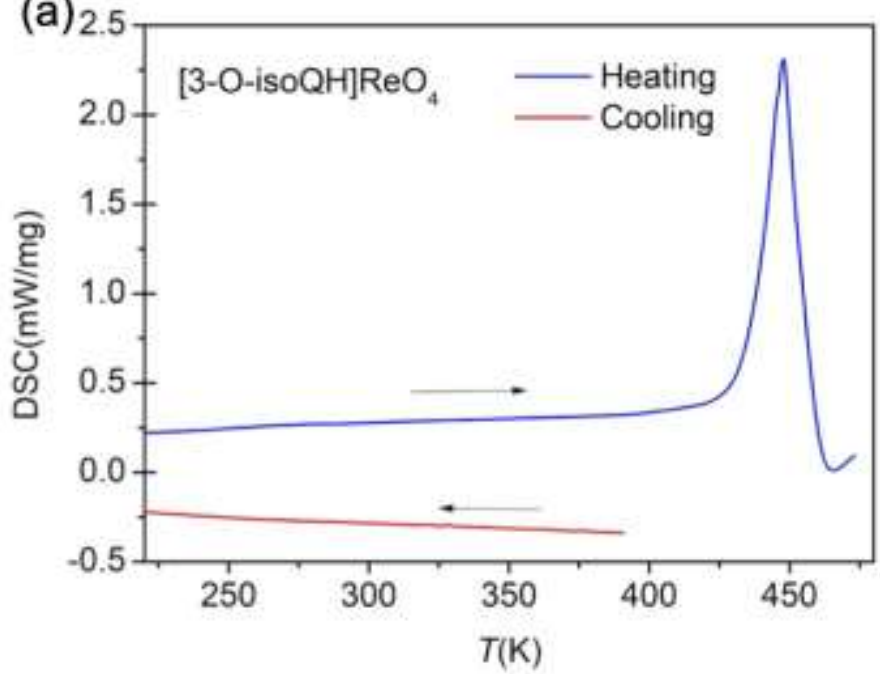

(b)

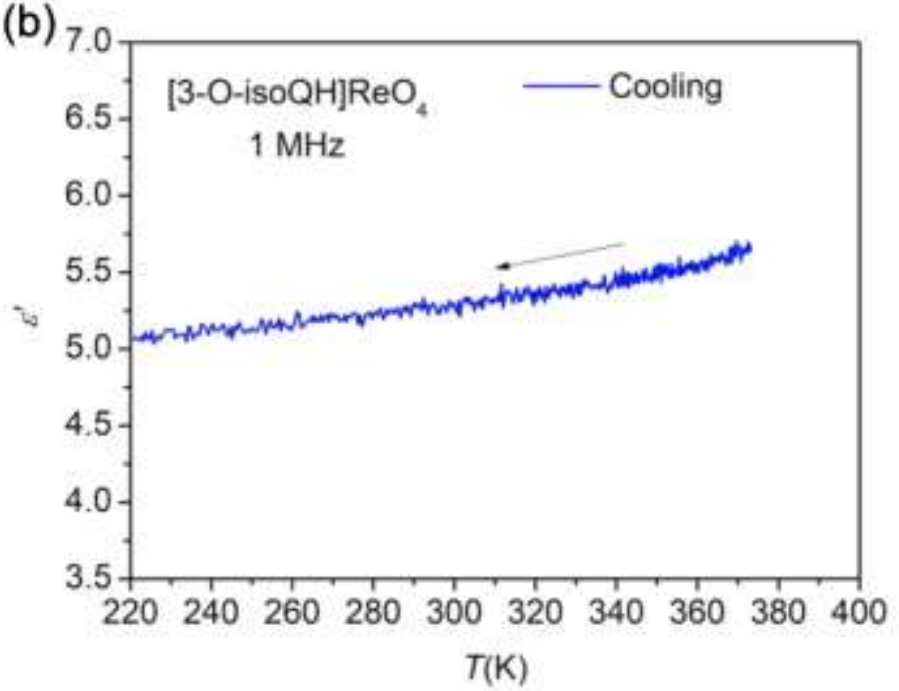

Figure S12. DSC measurement (a) and dielectric measurement(b) of [3-O-isoQH] $\mathrm{ReO}_{4}$. The sample of [3$\mathrm{O}$-isoQH] $\mathrm{ReO}_{4}$ in the heating run started to decompose at $433 \mathrm{~K}$. The cooling run was measured on another new sample of [3-O-isoQH] $\mathrm{ReO}_{4}$. 
(a)

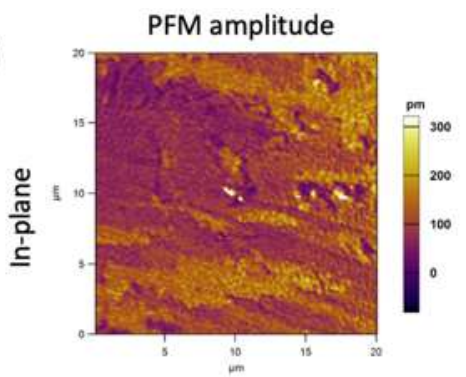

(b)

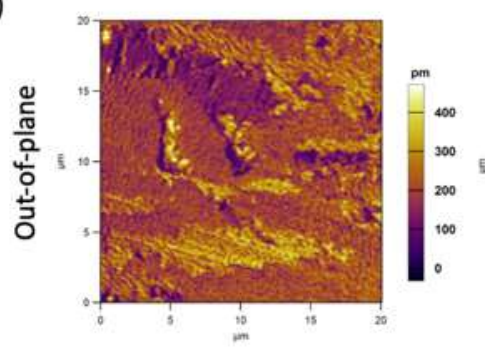

PFM phase

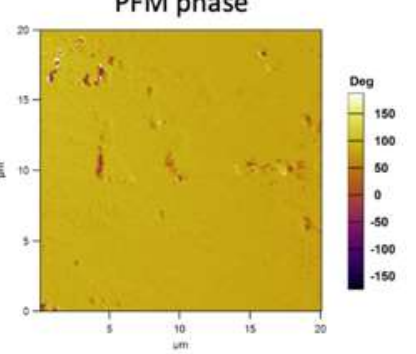

(c)
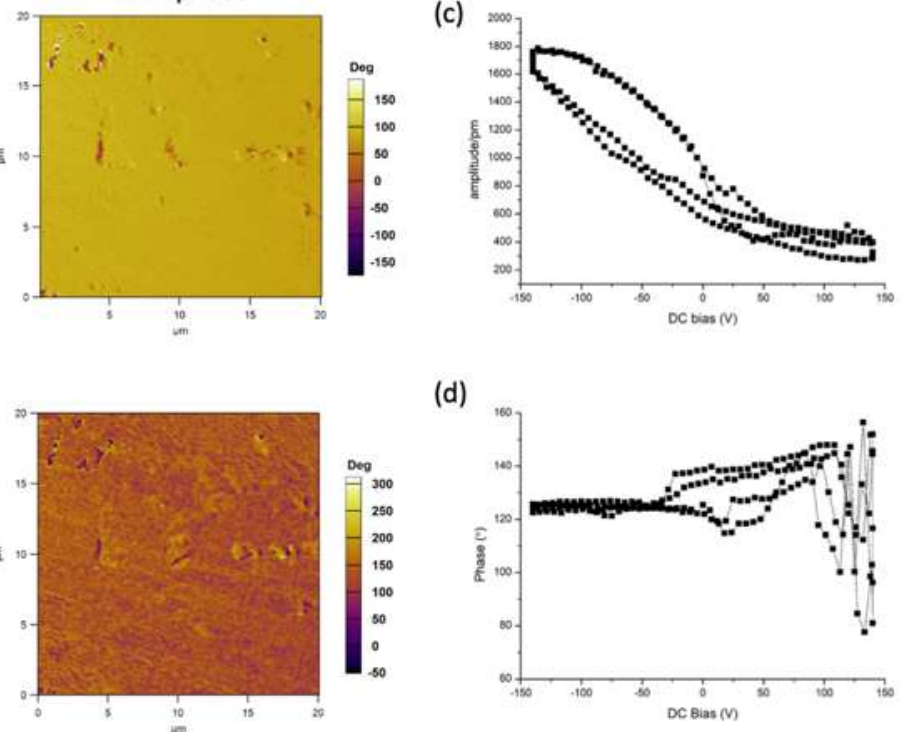

(d)

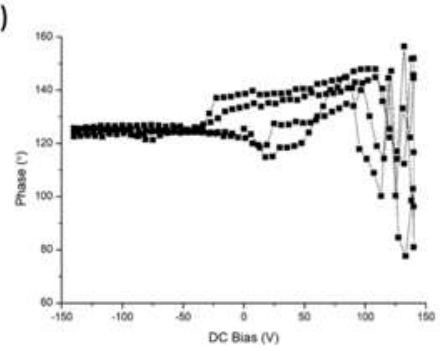

Figure S13. PFM amplitude (middle column), and PFM phase (right column) images of a $20 \times 20 \mu \mathrm{m}^{2}$ region at the initial state on the crystal of [isoQH] ReO4 $(0.6 \mathrm{~mm} \times 0.6 \mathrm{~mm} \times 0.5 \mathrm{~mm})($ In-plane (a), Out-of-plane(b)). PFM switching spectroscopy measurements (amplitude (a), pahse(b)) triggered by applied voltages on the crystal of $[\mathrm{isoQH}] \mathrm{ReO}_{4}$.

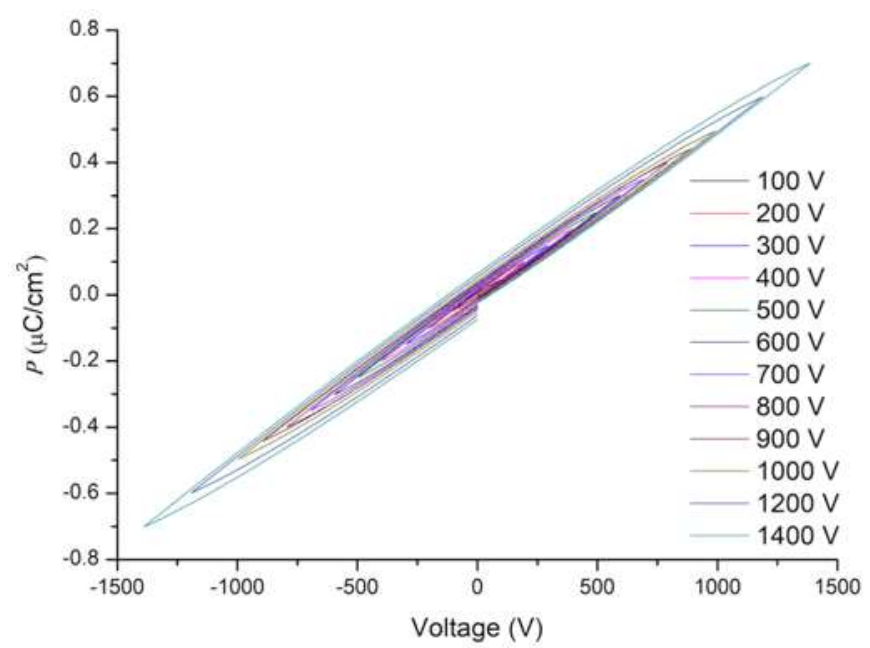

Figure S14. Polarization versus voltage hysteresis loop measurement of $[\mathrm{isoQH}] \mathrm{ReO}_{4}$ crystal $(1.0 \mathrm{~mm} \times 0.9$ $\mathrm{mm} \times 0.5 \mathrm{~mm})$ at room temperature. 
(a)

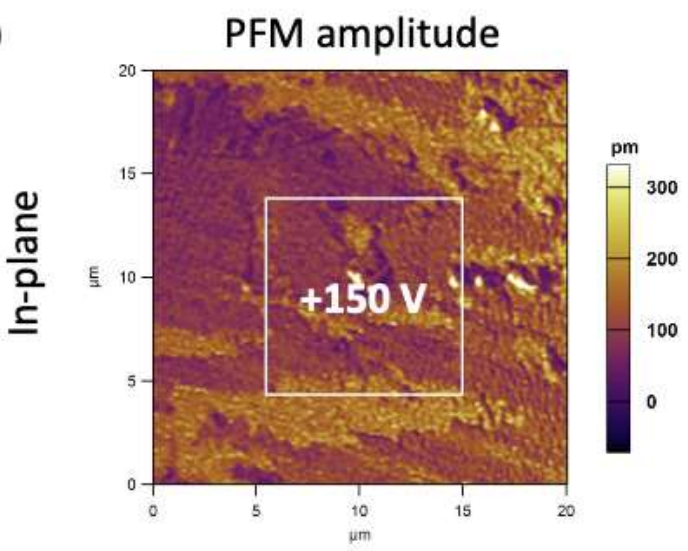

(b)

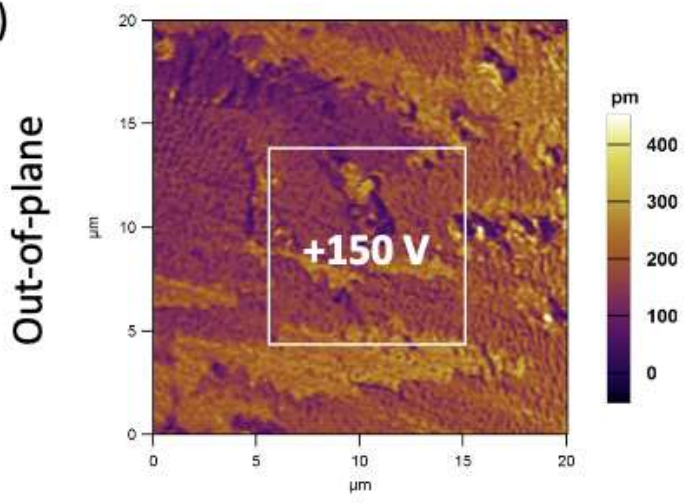

(c)

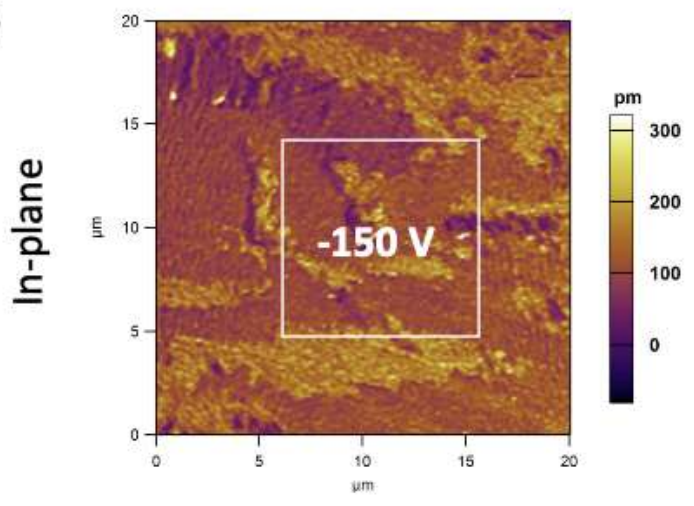

(d)

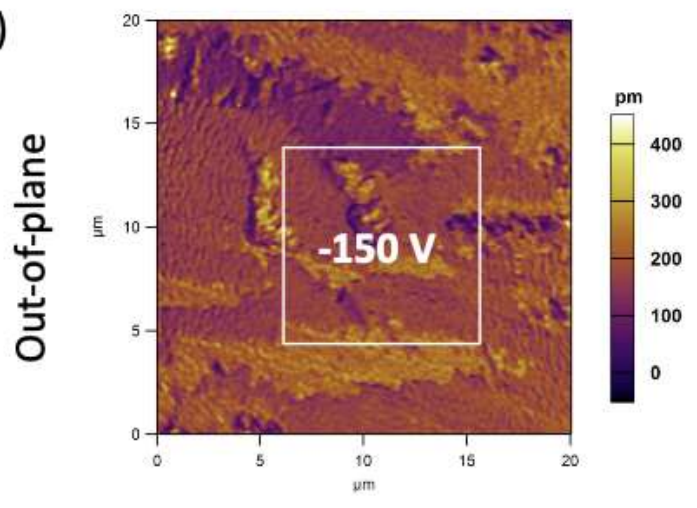

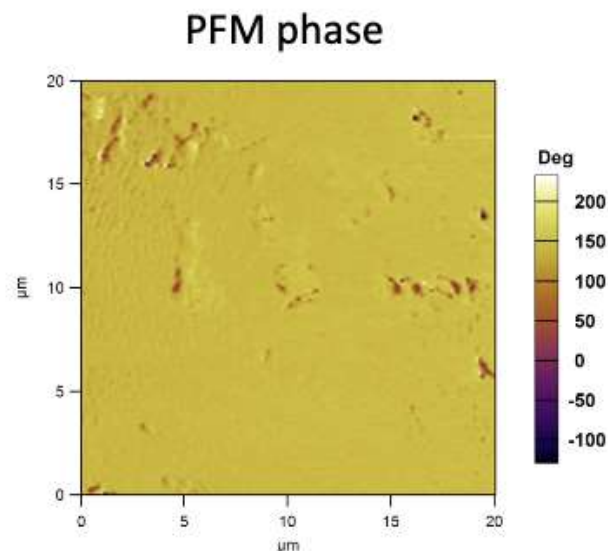
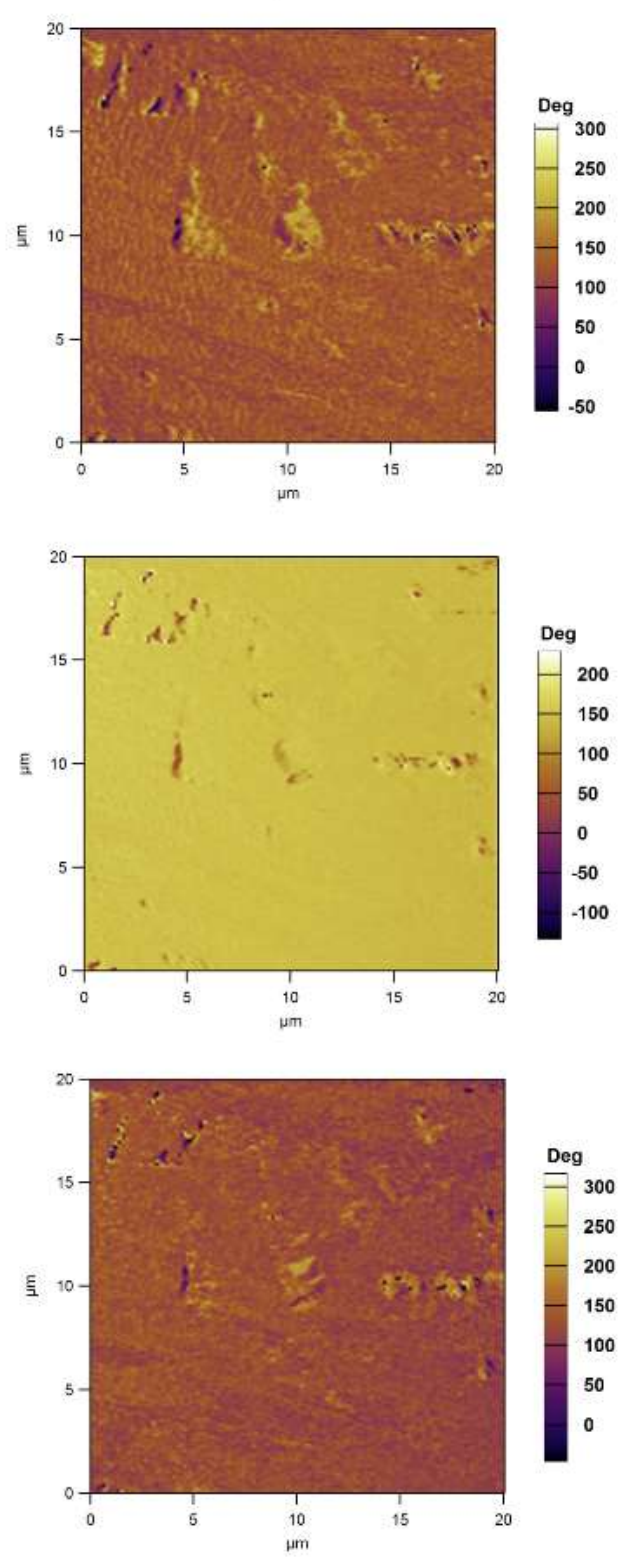

Figure S15. Vertical PFM amplitude (left column), and phase (right column) images of the crystal surface of [isoQH] $\mathrm{ReO}_{4}(0.6 \mathrm{~mm} \times 0.6 \mathrm{~mm} \times 0.5 \mathrm{~mm})$ by applying voltage (In-plane by positive voltage (a), Out-ofplane by positive voltage (b), In-plane by negative voltage (c), Out-of-plane by negative voltage (d)). 
Table S1 List of some metal-free organic-inorganic hybrid phase transition materials.

\begin{tabular}{|c|c|c|c|c|}
\hline Formula & $T_{\mathrm{c}}(\mathrm{K})$ & Symmetry Changes & Year & Ref \\
\hline Tri-glycine sulfate (TGS) & 322 & $2 \leftrightarrow 2 / \mathrm{m}$ & 1957 & 1 \\
\hline Triglycine fluoberyllate (TGFB) & 352 & $2 \leftrightarrow 2 / \mathrm{m}$ & 1973 & 2 \\
\hline Pyridinium perrhenate & 333 & $\mathrm{~mm} 2 \leftrightarrow-$ & 1997 & 3 \\
\hline Hdabco perchlorate & 377 & $\mathrm{~mm} 2 \leftrightarrow 4 / \mathrm{mmm}$ & 1999,2016 & 4 \\
\hline Hdabco tetrafluoroborate & 374 & $\mathrm{~mm} 2 \leftrightarrow 4 / \mathrm{mmm}$ & 1999, 2017 & 5 \\
\hline Hdabco perrhenate & $377, \mathbf{5 0 0}$ & $\mathrm{m} \leftrightarrow 4 \mathrm{~mm} \leftrightarrow \mathrm{m} \overline{3} \mathrm{~m}$ & 2017 & 6 \\
\hline Guanidinium perchlorate & 454 & $3 \mathrm{~m} \leftrightarrow \mathrm{m} \overline{3} \mathrm{~m}$ & 2011,2017 & 7 \\
\hline Tetraethylammonium perchlorate & 378 & $\mathrm{~m} \leftrightarrow \mathrm{m} \overline{3} \mathrm{~m}$ & 2016 & 8 \\
\hline Quinuclidinium periodate & 322 & $\mathrm{~mm} 2 \leftrightarrow \mathrm{m} \overline{3} \mathrm{~m}$ & 2017 & 9 \\
\hline Quinuclidinium perrhenate & 345,367 & $\mathrm{~mm} 2 \leftrightarrow 3 \mathrm{~m} \leftrightarrow \mathrm{m} \overline{3} \mathrm{~m}$ & 2017 & 10 \\
\hline$[\mathrm{D}-55 \mathrm{DMBP}][\mathrm{Hia}]$ & 268 & $1 \leftrightarrow \overline{1}$ & 2007 & 11 \\
\hline$[\mathrm{D}-55 \mathrm{DMBP}][\mathrm{Dia}]$ & 335 & $1 \leftrightarrow \overline{1}$ & 2007 & 11 \\
\hline$[\mathrm{H}-\mathrm{dppz}][\mathrm{Hca}]$ & 402 & $\mathrm{~m} \leftrightarrow 2 / \mathrm{m}$ & 2013 & 12 \\
\hline Diisopropylammonium Chloride & 440 & $2 \leftrightarrow 2 / \mathrm{m}$ & 2011 & 13 \\
\hline Diisopropylammonium Bromide & 426 & $2 \leftrightarrow 2 / \mathrm{m}$ & 2013 & 14 \\
\hline$[\mathrm{Me}-\mathrm{dabco}]-\mathrm{NH}_{4} \mathrm{I}_{3}$ & 448 & $3 \leftrightarrow 432$ & 2018 & 15 \\
\hline Cyclohexylammonium-(18- & 390 & $\mathrm{~mm} 2 \leftrightarrow \mathrm{mmm}$ & 2015 & 16 \\
\hline Cyclohexylammonium-(18- & 397 & $\mathrm{~mm} 2 \leftrightarrow \mathrm{mmm}$ & 2015 & 16 \\
\hline$(R)-(-)-3$ hydroxlyquinuclidinium & 340 & $4 \leftrightarrow 432$ & 2016 & 17 \\
\hline Pyridinium periodate & 321 & $\mathrm{~mm} 2 \leftrightarrow \mathrm{mmm}$ & 2001 & 18 \\
\hline pyrazine $\mathrm{HBF}_{4}$ & 349,356 & $\mathrm{mmm} \leftrightarrow 2 / \mathrm{m} \leftrightarrow 2 / \mathrm{m}$ & 2006 & 19 \\
\hline 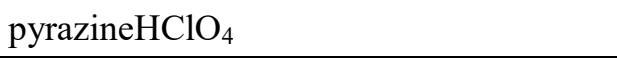 & 355,358 & $\mathrm{mmm} \leftrightarrow-$ & 2006 & 19 \\
\hline guanidinium iodide & 349 & $6 \mathrm{~mm} \leftrightarrow 2 / \mathrm{m}$ & 2013 & 20 \\
\hline Imidazolium periodate & 310 & $2 \leftrightarrow 2 / \mathrm{m}$ & 2014 & 21 \\
\hline Imidazolium perchlorate & 373 & $3 \mathrm{~m} \leftrightarrow \overline{3} \mathrm{~m}$ & 2014 & 22 \\
\hline$\left(\right.$ dabcoH$\left._{2}\right)\left(\mathrm{BF}_{4}\right)_{2} \cdot \mathrm{H}_{2} \mathrm{O}$ & 234 & $\mathrm{~mm} 2 \leftrightarrow \mathrm{mm} 2$ & 2016 & 23 \\
\hline$\left(\right.$ dabcoH$\left._{2}\right)\left(\mathrm{ClO}_{4}\right)_{2} \cdot \mathrm{H}_{2} \mathrm{O}$ & 248 & $\mathrm{~mm} 2 \leftrightarrow \mathrm{mm} 2$ & 2016 & 23 \\
\hline$\left(\right.$ dabcoH$\left._{2}\right) \mathrm{H}_{3} \mathrm{O}\left(\mathrm{BF}_{4}\right)_{3}$ & 335 & $6 / \mathrm{m} \leftrightarrow 6 / \mathrm{mmm}$ & 2018 & 24 \\
\hline$\left(\right.$ dabcoH$\left._{2}\right) \mathrm{H}_{3} \mathrm{O}\left(\mathrm{ClO}_{4}\right)_{3}$ & $134,295.8$, & $-\leftrightarrow 23 \leftrightarrow \mathrm{m} \overline{3}$ & 2018 & 24 \\
\hline 1-azabicyclo[2.2.1]heptanium perrhenate & 199,322 & $3 \mathrm{~m} \leftrightarrow \mathrm{m} \overline{3} \mathrm{~m}$ & 2019 & 25 \\
\hline 1-azabicyclo[2.2.1]heptanium periodate & 244,258 & $-\leftrightarrow \mathrm{m} \overline{3} \mathrm{~m}$ & 2019 & 25 \\
\hline$[9-\operatorname{tacn}-3] \mathrm{Cl}_{2}\left[\mathrm{BF}_{4}\right]$ & 400 & $2 \leftrightarrow 6 / \mathrm{mmm}$ & 2019 & 26 \\
\hline [3-oxoquinuclidinium] $\mathrm{ClO}_{4}$ & 457 & $\mathrm{~mm} 2 \leftrightarrow \mathrm{m} \overline{3} \mathrm{~m}$ & 2019 & 27 \\
\hline [isoQ] $\mathrm{ReO}_{4}$ & 393 & $2 \leftrightarrow 4 / \mathrm{m}$ & This work & \\
\hline [HDABCO][TFSA] & 274.3 & $2 / \mathrm{m} \leftrightarrow 2$ & 2020 & 28 \\
\hline [DDABCO][TFSA] & 327.4 & $2 / \mathrm{m} \leftrightarrow 2$ & 2020 & 28 \\
\hline [3.2.1-dabco] $\mathrm{BF}_{4}$ & 357 & $\mathrm{~mm} 2 \leftrightarrow-$ & 2020 & 29 \\
\hline dabco sulfamate & 149 & $2 / \mathrm{m} \leftrightarrow 2 / \mathrm{m}$ & 2020 & 30 \\
\hline$\left(\mathrm{C}_{8} \mathrm{H}_{20} \mathrm{~N}\right)\left[\mathrm{BF}_{4}\right]$ & 257 & $2 / \mathrm{m} \leftrightarrow-$ & 2020 & 31 \\
\hline$[2,4,6$-trimethylpyridinium $]\left[\mathrm{BF}_{4}\right]$ & 241 & $\mathrm{~m} \leftrightarrow \mathrm{mm} 2$ & 2011 & 32 \\
\hline
\end{tabular}


Table S2 Crystal data and structure refinements of compound [N-Me-isoQH] $\mathrm{ReO}_{4}$

\section{Crystal Data}

\begin{tabular}{|c|c|c|c|c|}
\hline Compounds & & & $\mathrm{H}] \mathrm{ReO}_{4}$ & \\
\hline Empirical formula & & & ${ }_{4} \operatorname{Re}$ & \\
\hline Temperature(K) & 173 & 273 & 300 & 333 \\
\hline Formula Weight & 376.43 & 376.43 & 376.43 & 376.43 \\
\hline Crystal system & trigonal & trigonal & trigonal & trigonal \\
\hline Space group & $R \overline{3}$ & $R \overline{3}$ & $R \overline{3}$ & $R \overline{3}$ \\
\hline$a[\AA]$ & $23.2456(5)$ & $23.2456(5)$ & $23.5948(5)$ & $23.4935(10)$ \\
\hline$b[\AA]$ & $23.2456(5)$ & $23.2456(5)$ & $23.5948(5)$ & $23.4935(10)$ \\
\hline$c[\AA]$ & $10.5507(3)$ & $10.5507(3)$ & $10.6826(3)$ & $10.6077(7)$ \\
\hline$\alpha\left[^{\circ}\right]$ & 90 & 90 & 90 & 90 \\
\hline$\beta\left[^{\circ}\right]$ & 90 & 90 & 90 & 90 \\
\hline$\gamma\left[^{\circ}\right]$ & 120 & 120 & 120 & 120 \\
\hline Volume $[\AA]^{3}$ & $4937.3(3)$ & 4937.3(3) & $5150.4(3)$ & $5070.5(5)$ \\
\hline Z & 18 & 18 & 18 & 18 \\
\hline Reflns measured & 4739 & 5755 & 15579 & 5811 \\
\hline GOF & 1.011 & 1.020 & 1.019 & 1.055 \\
\hline$R_{1}[I>2 \sigma(I)]$ & 0.0426 & 0.0395 & 0.0743 & 0.0696 \\
\hline $\mathrm{w} R_{2}[I>2 \sigma(I)]$ & 0.1150 & 0.0929 & 0.2050 & 0.1970 \\
\hline
\end{tabular}




\section{References and Notes}

1. Hoshino, S.; Mitsui, T.; Jona, F.; Pepinsky, R. Dielectric and thermal study of triglycine sulfate and triglycine fluoberyllate. Physical Review 1957, 107, 1255-1258.

2. Mercado, A.; Gonzalo, Julio A. Ferroelectric behavior of triglycine fluoroberyllate near Tc. Physical Review B: Solid State 1973 7, (7), 30743079 .

3. Wasicki, J.; Czarnecki, P.; Pajak, Z.; Nawrocik, W.; Szczepanski, W. Ferroelectric properties of pyridinium perrhenate. Journal of Chemical Physics 1997, 107, (2), 576-579.

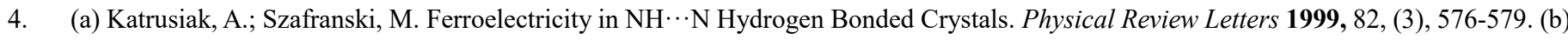
Tang, Y.-Y. Z., Wan-Ying; Li, P. F.; Ye, H. Y.; You, Y. M.; Xiong, R. G. Ultrafast Polarization Switching in a Biaxial Molecular Ferroelectric Thin Film: [Hdabco]ClO4. Journal of the American Chemical Society 2016, 138, (48), 15784- 15789.

5. (a) Katrusiak, A.; Szafranski, M. Ferroelectricity in NH $\cdots$ N Hydrogen Bonded Crystals. Physical Review Letters 1999, 82, (3), 576-579. (b)Shi, P.-P.; Tang, Y. Y.; Li, P. F.; Ye, H. Y.; Xiong, R. G. De Novo Discovery of [Hdabco]BF4 Molecular Ferroelectric Thin Film for Nonvolatile Low-Voltage Memories. Journal of the American Chemical Society 2017, 139, (3), 1319-1324.

6. Tang, Y.-Y.; Li, P. F.; Zhang, W. Y.; Ye, H. Y.; You, Y. M.; Xiong, R. G. A multiaxial molecular ferroelectric with highest Curie temperature and fastest polarization switching. Journal of the American Chemical Society 2017, 139, (39), 13903- 13908.

7. (a) Szafranski, M. Simple Guanidinium Salts Revisited: Room-Temperature Ferroelectricity in Hydrogen-Bonded Supramolecular Structures. Journal of Physical Chemistry B 2011, 115, (27), 8755- 8762. (b) Pan, Q.; Liu, Z. B.; Zhang, H. Y.; Zhang, W. Y.; Tang, Y. Y.; You, Y. M.; Li, P. F.; Liao, W. Q.; Shi, P. P.; Ma, R. W.; Wei, R. Y.; Xiong, R. G. A Molecular Polycrystalline Ferroelectric with Record-High Phase Transition Temperature Advanced Materials (Weinheim, Germany) 2017, 29, (29).

8. Ye, H. Y.; Ge, J. Z.; Tang, Y. Y.; Li, P. F.; Zhang, Y.; You, Y. M; Xiong, R. G. Molecular Ferroelectric with Most Equivalent Polarization Directions Induced by the Plastic Phase Transition Journal of the American Chemical Society 2016, 138, (40), $13175-13178$.

9. You, Y. M.; Tang, Y. Y.; Li, P. F.; Zhang, H. Y.; Zhang, W. Y.; Zhang, Y.; Ye, H. Y.; Nakamura, T.; Xiong, R. G. Quinuclidinium salt ferroelectric thin-film with duodecuple-rotational polarization-directions. Nature Communications 2017, 8, 14934.

10. Tang, Y. Y.; Li, P. F.; Shi, P. P.; Zhang, W. Y.; Wang, Z. X. ; You, Y. M.; Ye, H. Y.; Nakamura, T.; Xiong, R. G. Visualization of roomtemperature ferroelectricity and polarization rotation in the thin film of quinuclidinium perrhenate Physical Review Letters 2017, 119, (20), 207602/1- 207602/6.

11. Horiuchi, S.; Kumai, R.; Tokura, Y. A supramolecular ferroelectric realized by collective proton transfer. Angewandte Chemie, International Edition 2007, 46, (19), 3497- 3501.

12. Horiuchi, S.; Kumai, R.; Tokura, Y. High-Temperature and Pressure-Induced Ferroelectricity in Hydrogen-Bonded Supramolecular Crystals of Anilic Acids and 2,3-Di(2-pyridinyl)pyrazine. Journal of the American Chemical Society 2013, 135, (11), 4492-4500.

13. Fu, D. W.; Zhang, W.; Cai, H. L.; Ge, J. Z.; Zhang, Y.; Xiong, R. G. Diisopropylammonium Chloride: A Ferroelectric Organic Salt with a High Phase Transition Temperature and Practical Utilization Level of Spontaneous Polarization. Advanced Materials (Weinheim, Germany) 2011, $23,(47), 5658-5662$.

14. Fu, D. W.; Cai, H. L.; Liu, Y.; Ye, Q.; Zhang, W.; Zhang, Y.; Chen, X. Y.; Giovannetti, G.; Capone, M.; Li, J. Y.; Xiong, R. G. Diisopropylammonium Bromide Is a High-Temperature Molecular Ferroelectric Crystal. Science 2013, 339, 425 - 428.

15. Ye, H. Y.; Tang, Y. Y.; Li, P. F.; Liao, W. Q.; Gao, J. X.; Hua, X. N. Metal-free three-dimensional perovskite ferroelectrics. Science 2018, $361,151-155$

16. Tang, Y. Z.; Yu, Y. M.; Xiong, J. B.; Tan, Y. H.; Wen, H. R. Unusual High-Temperature Reversible Phase-Transition Behavior, Structures, and Dielectric-Ferroelectric Properties of Two New Crown Ether Clathrates. Journal of the American Chemical Society 2015, 137, (41), 1334513351.

17. Li, P. F.; Tang, Y. Y.; Wang, Z. X.; Ye, H. Y.; You, Y. M.; Xiong, R. G. Anomalously rotary polarization discovered in homochiral organic ferroelectrics. Nature Communications 2016, 7, 13635.

18. Maluszynska, H.; Czarnecki, P.; Lewicki, S.; Wasicki, J.; Gdaniec, M. Structure and dynamics of ferroelectric pyridinium periodate. Journal of Physics: Condensed Matter 2001, 13, (48), 11053-11065. 


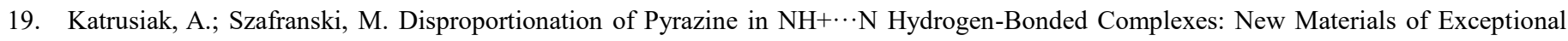
Dielectric Response. Journal of the American Chemical Society 2006, 128, (49), 15775-155785.

20. Szafranski, M.; Jarek, M. Origin of spontaneous polarization and reconstructive phase transition in guanidinium iodide. CrystEngComm 2013, 15, (23), 4617-4623.

21. Zhang, Y.; Ye, H. Y.; Cai, H. L.; Fu, D. W.; Ye, Q.; Zhang, W.; Zhou, Q. H.; Wang, J. L.; Yuan, G. L.; Xiong, R. G. Switchable Dielectric, Piezoelectric, and Second-Harmonic Generation (SHG) Bistability in a New Improper Ferroelectric above Room Temperature. Advanced Materials (Weinheim, Germany) 2014, 26, (26), 4515-4520.

22. Zhang, Y.; Liu, Y. M.; Ye, H. Y.; Fu, D. W.; Gao, W. X.; Ma, H.; Liu, Z. G.; Liu, Y. Y.; Zhang, W.; Li, J. Y.; Yuan, G. L.; Xiong, R. G. A Molecular Ferroelectric Thin Film of Imidazolium Perchlorate That Shows Superior Electromechanical Coupling. Angewandte Chemie, International Edition 2014, 53, (20), 5064-5068.

23. Han, X. B.; Hu, P.; Shi, C.; Zhang, W. 1,4-Diazabicyclo[2.2.2]octane-based disalts showing non-centrosymmetric structures and phase transition behaviors. CrystEngComm 2016, 18, (9), 1563-1569.

24. Szafranski, M. Synthesis, Crystal Structures, and Phase Transitions of Dabco Oxonium Triperchlorate and Tritetrafluoroborate. Crystal Growth \& Design 2018, 18, (11), 7106- 7113.

25. Harada, J.; Kawamura, Y.; Takahashi, Y.; Uemura, Y.; Hasegawa, T.; Taniguchi, H.; Maruyama, K. Plastic/ferroelectric crystals with easily switchable polarization: low-voltage operation, unprecedentedly high pyroelectric performance, and large piezoelectric effect in polycrystalline forms. Journal of the American Chemical Society 2019, 141, (23), 9349-9357.

26. Gao, J. X.; Hua, X. N.; Chen, X. G.; Liao, W. Q. A high temperature optic-electric duple switching organic ionic compound: 1,4,7triazoniacyclononane tetrafluoroborate dichloride. Journal of Materials Chemistry C: Materials for Optical and Electronic Devices 2019, 7, (18), 5348-5352.

27. Yang, C. K.; Chen, W. N.; Ding, Y. T.; Wang, J.; Rao, Y.; Liao, W. Q.; Xie, Y. F.; Zou, W. N.; Xiong, R. G. Directional intermolecular interactions for precise molecular design of a high-Tc multiaxial molecular ferroelectric Journal of the American Chemical Society 2019, 141, (4), $1781-1788$.

28. Song, X. J.; Zhang, Z. X.; Chen, X. G.; Zhang, H. Y.; Pan, Q.; Yao, J.; You, Y. M.; Xiong, R. G. Bistable state of protons for low-voltage memories Journal of the American Chemical Society 2020, 142, (19), 9000- 9006.

29. Wei, Z. H.; Jiang, Z. T.; Zhang, X. X.; Li, M. L.; Tang, Y. Y.; Chen, X. G.; Cai, H.; Xiong, R. G. Rational Design of Ceramic-Like Molecular Ferroelectric by Quasi-Spherical Theory. Journal of the American Chemical Society (2020), 142(4), 1995-2000. 2020, 142, (4), 1995-2000.

30. Szafranski, M., Large Negative Linear Compressibility Triggered by Hydrogen Bonding. Journal of Physical Chemistry C 2020, 124, (21), $11631-11638$.

31. Moskwa, M.; Ganczar, E.; Sobieszczyk, P.; Medycki, W.; Zielinski, P.; Jakubas, R.; Bator, G. Temperature-Stimulus Responsive Ferroelastic Molecular-Ionic Crystal: $\left(\mathrm{C}_{8} \mathrm{H}_{20} \mathrm{~N}\right)\left[\mathrm{BF}_{4}\right]$. Journal of Physical Chemistry C 2020, 124, (33), 18209-18218.

32. Maciej, W.; Andrzej, B.; Ryszard, J. Organic-inorganic compounds with strong nonlinear optical properties based on 2,4,6trimethylpyridinium and tetrahedral BF4- networks. Physical Review B: Condensed Matter and Materials Physics 2011, 83, (14), 144103/1$144103 / 13$ 
NMR spectra

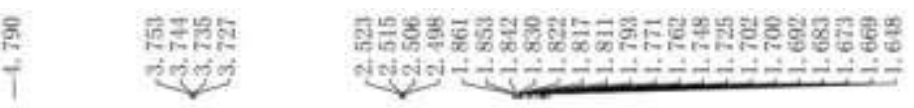<smiles>O=C1NC2CCC1C2</smiles>

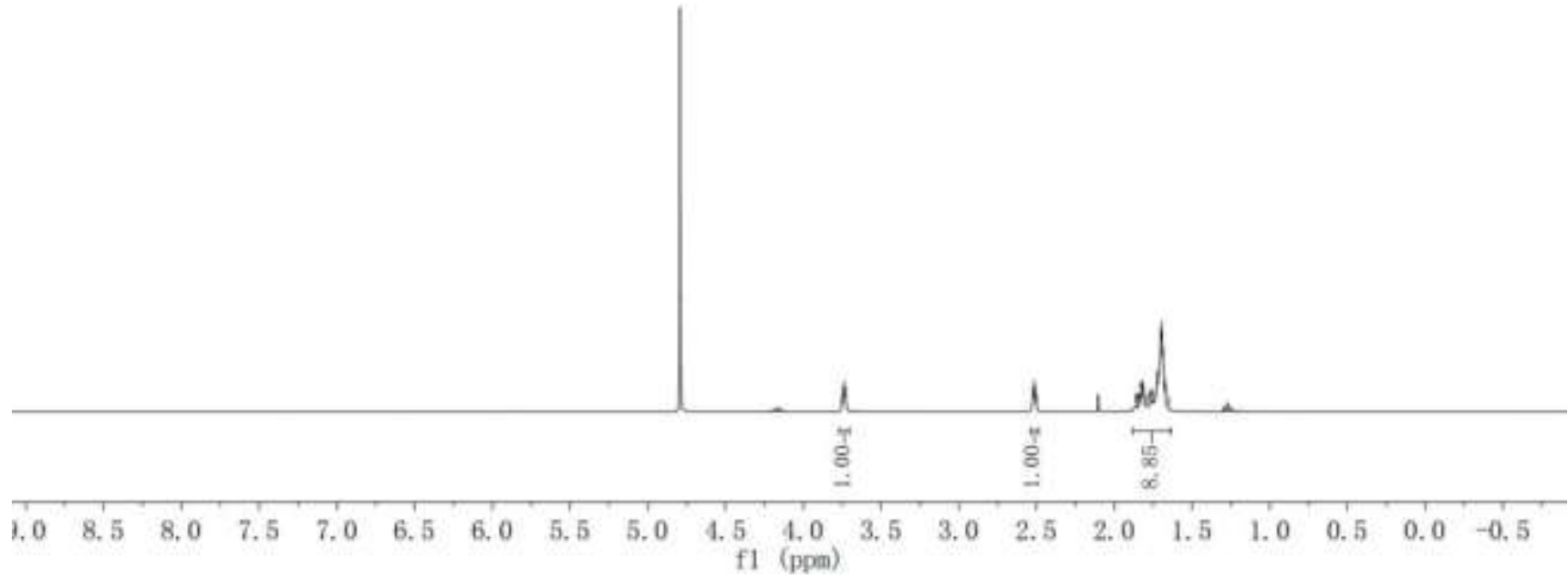<smiles>O=C1NC2CCC1C2</smiles> 
$2 \int_{\mathrm{OH}_{2}}^{\mathrm{O}}\left[\mathrm{ReO}_{4}\right]^{-}$
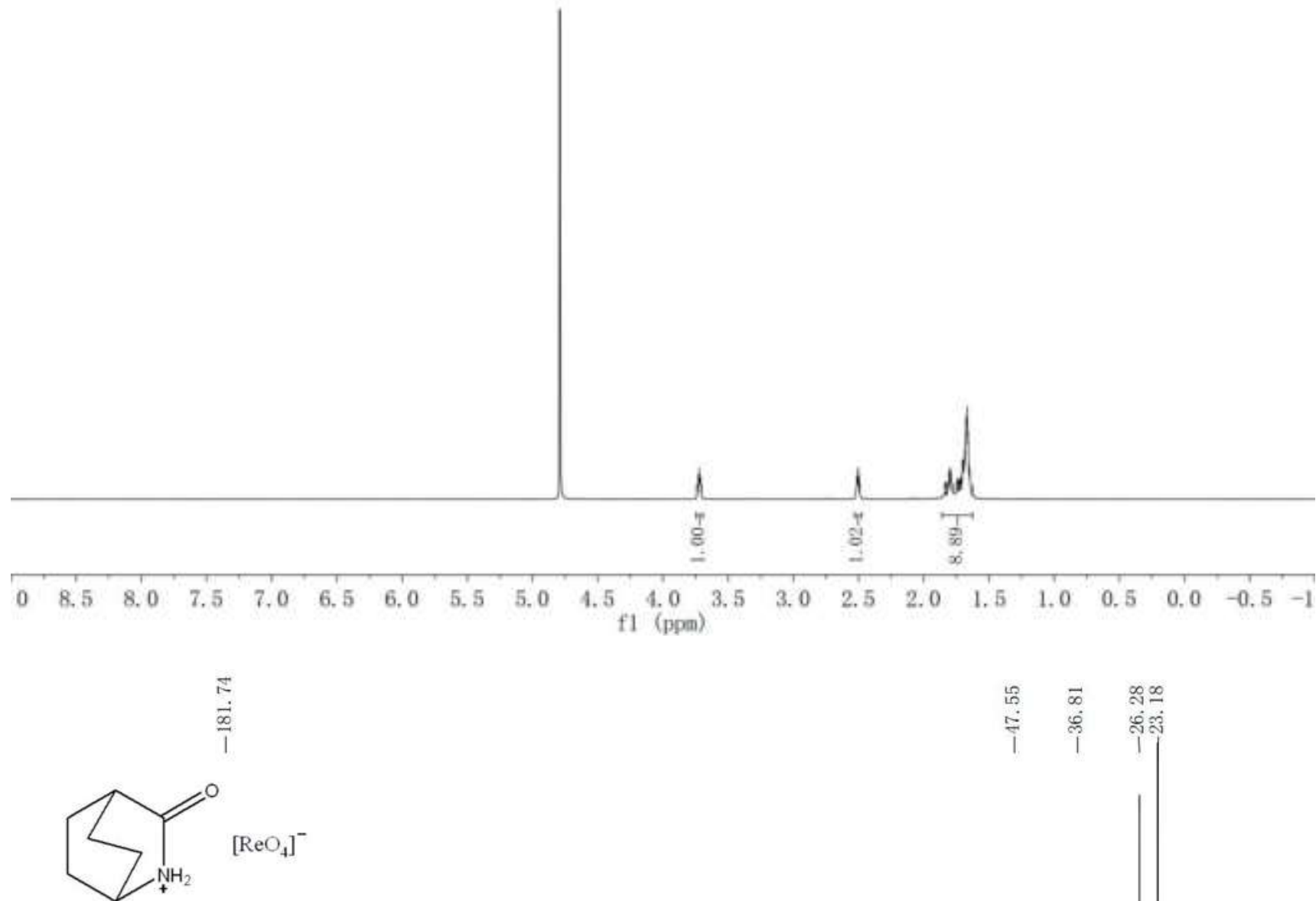

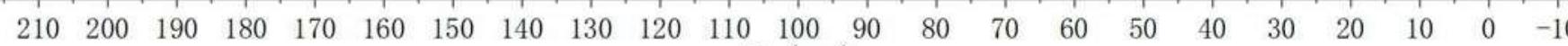
f1 (ppm) 

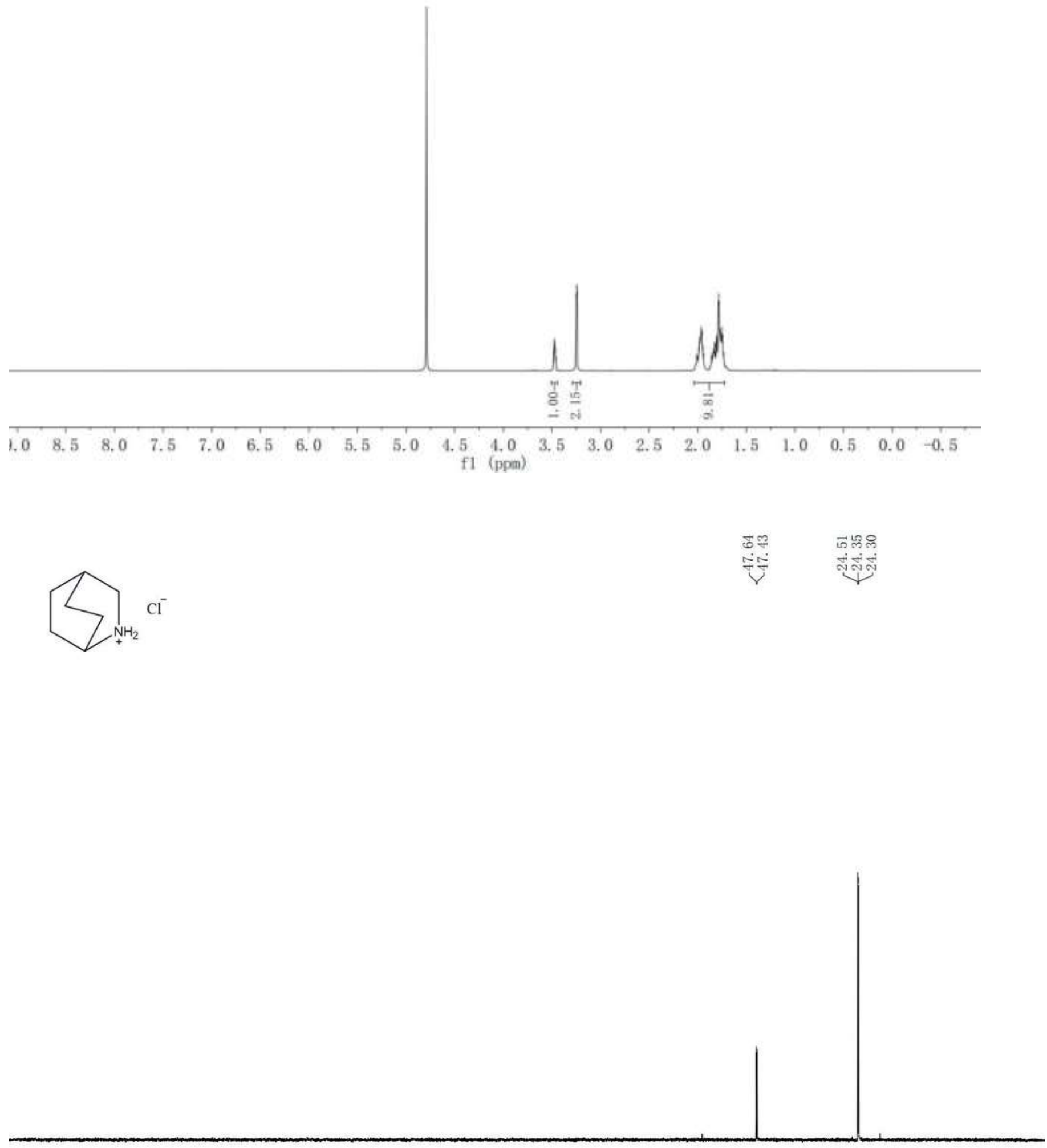

$\begin{array}{lllllllllllllllllllllll}210 & 200 & 190 & 180 & 170 & 160 & 150 & 140 & 130 & 120 & 110 & 100 & 90 & 80 & 70 & 60 & 50 & 40 & 30 & 20 & 10 & 0 & -10\end{array}$ 1 ( 15 
$\sum_{\mathrm{NH}_{2}}\left[\mathrm{ReO}_{4}\right]$

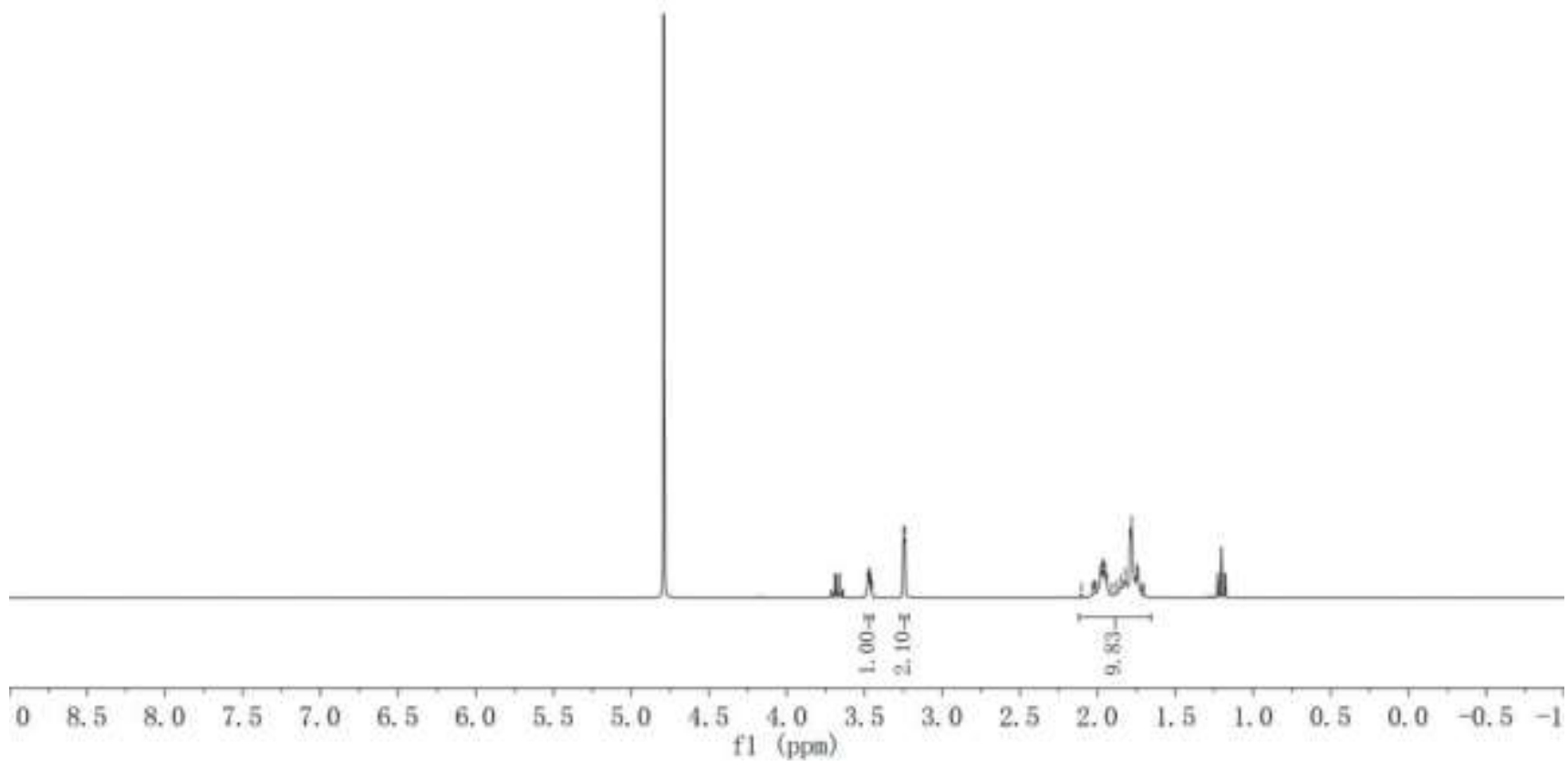

$\sum_{\mathrm{NH}_{2}}\left[\mathrm{ReO}_{4}\right]^{-}$

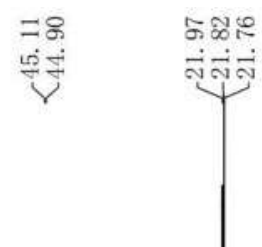

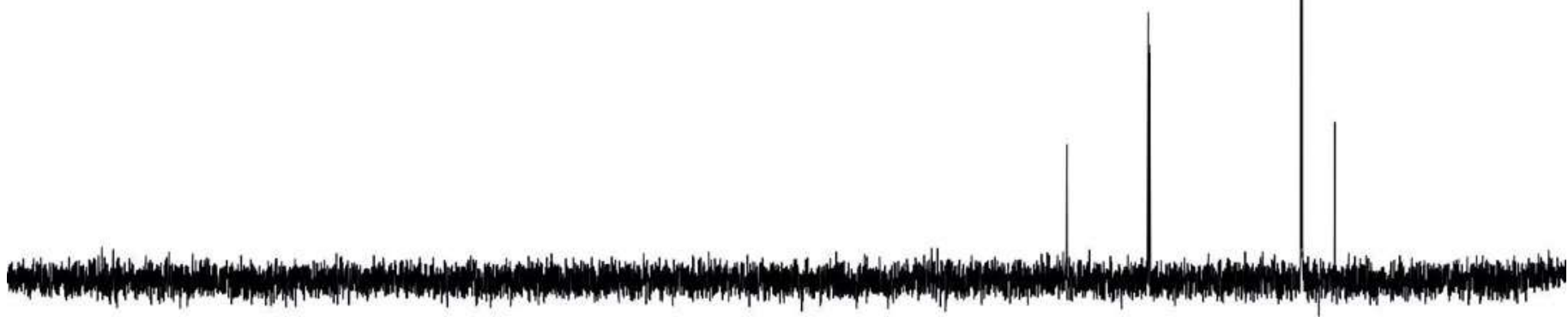

$\begin{array}{lllllllllllllllllllllll}210 & 200 & 190 & 180 & 170 & 160 & 150 & 140 & 130 & 120 & 110 \\ \mathrm{f} 1 & \begin{array}{c}100 \\ (\mathrm{ppm})\end{array} & 90 & 80 & 70 & 60 & 50 & 40 & 30 & 20 & 10 & 0 & -10\end{array}$


<smiles>O=C1C2CCC(C2)N1CN1CCCC1</smiles>

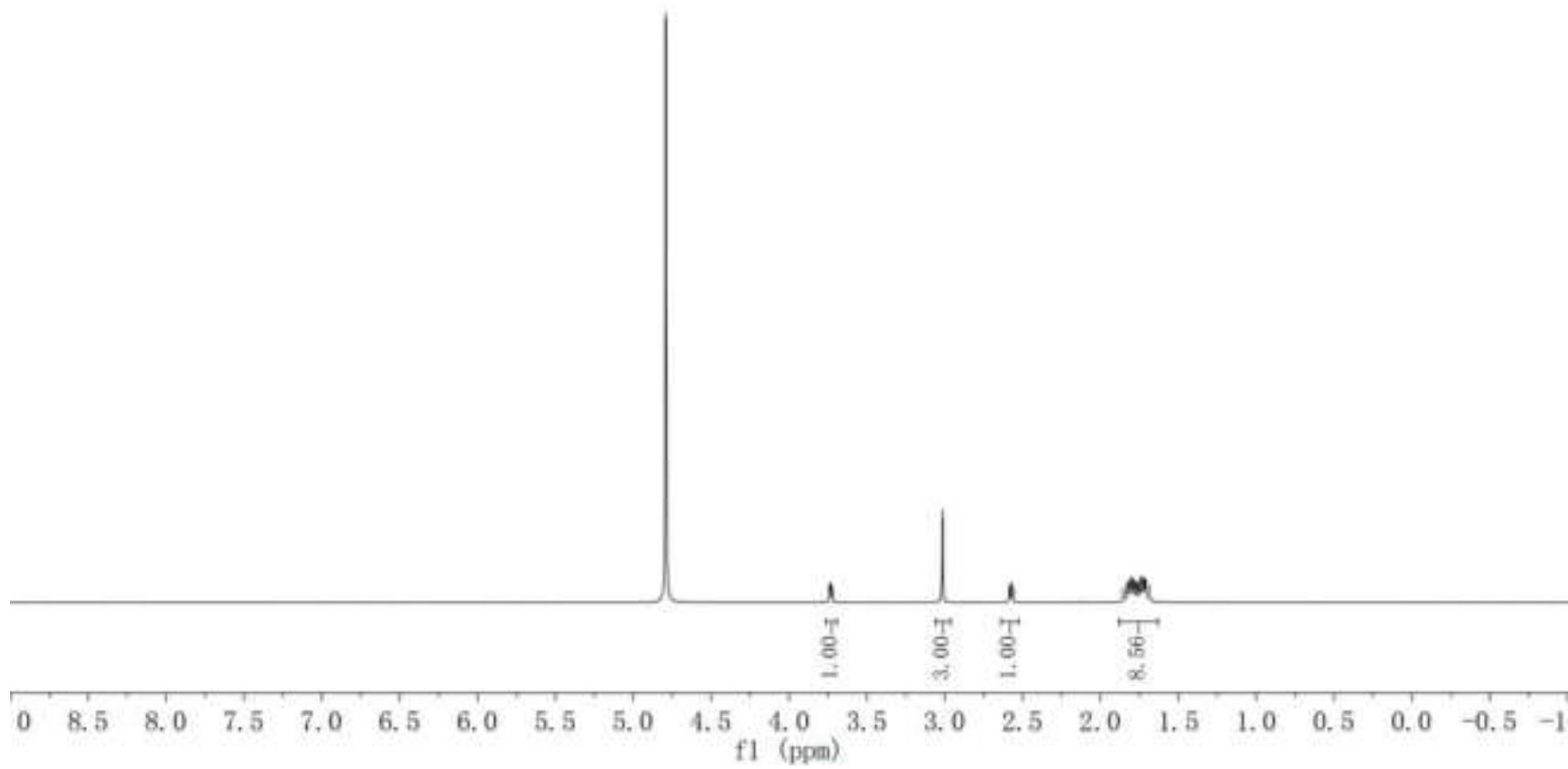<smiles>CN1CC2CCC(C2)C1=O</smiles>

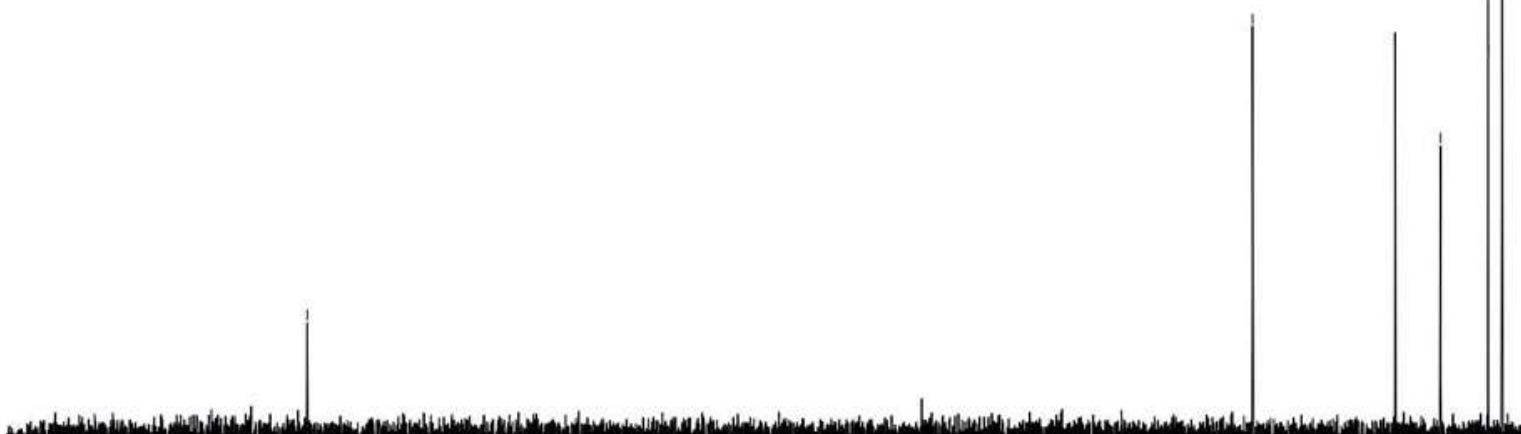

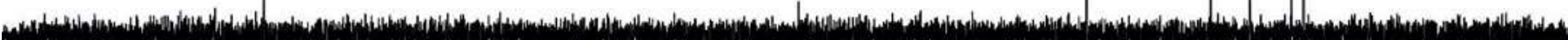

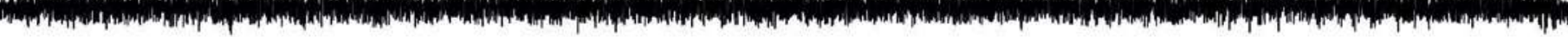

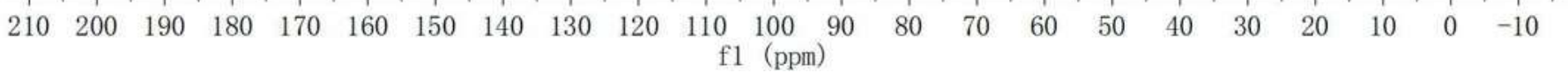




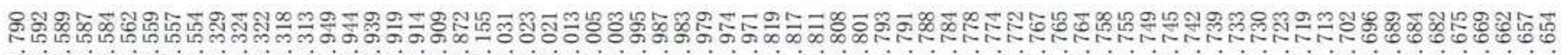

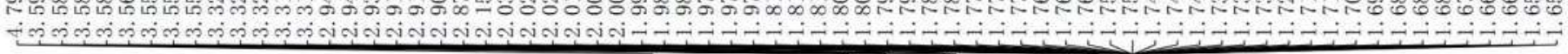
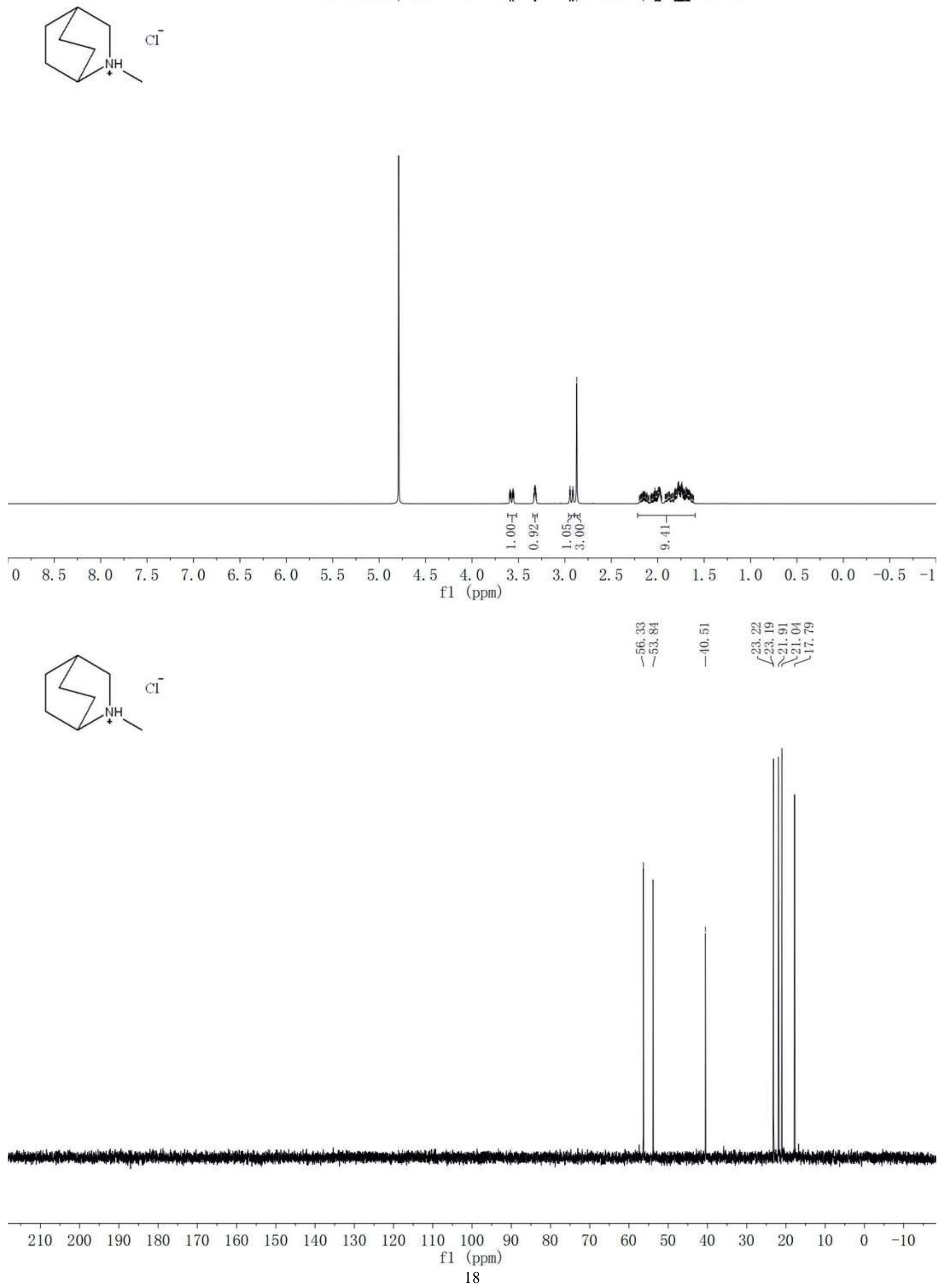
<smiles>C[NH+]1CC2CCC1[C@H]1CC21[O+]</smiles>

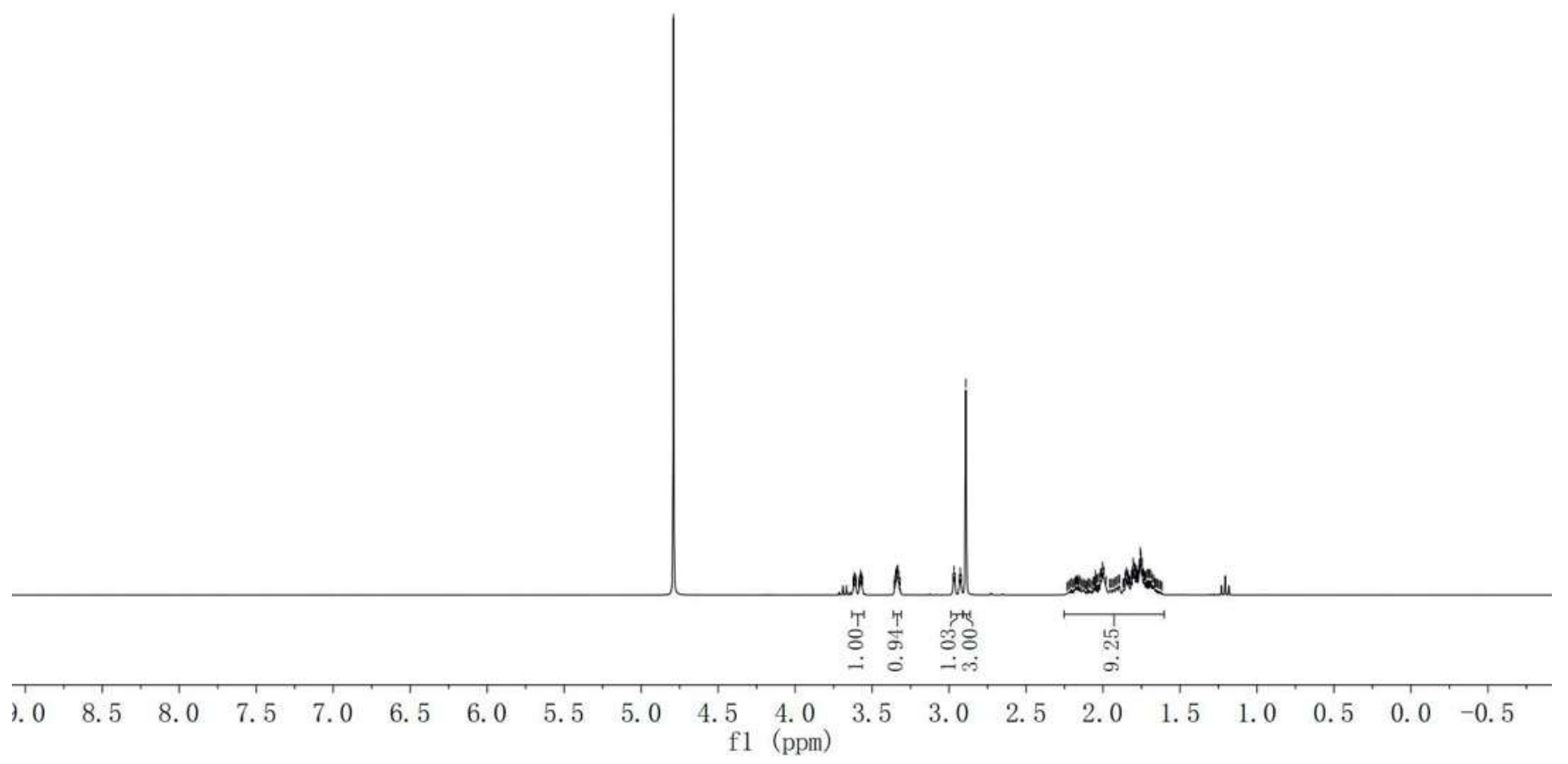<smiles>CNC1CC2CCC1C2</smiles>

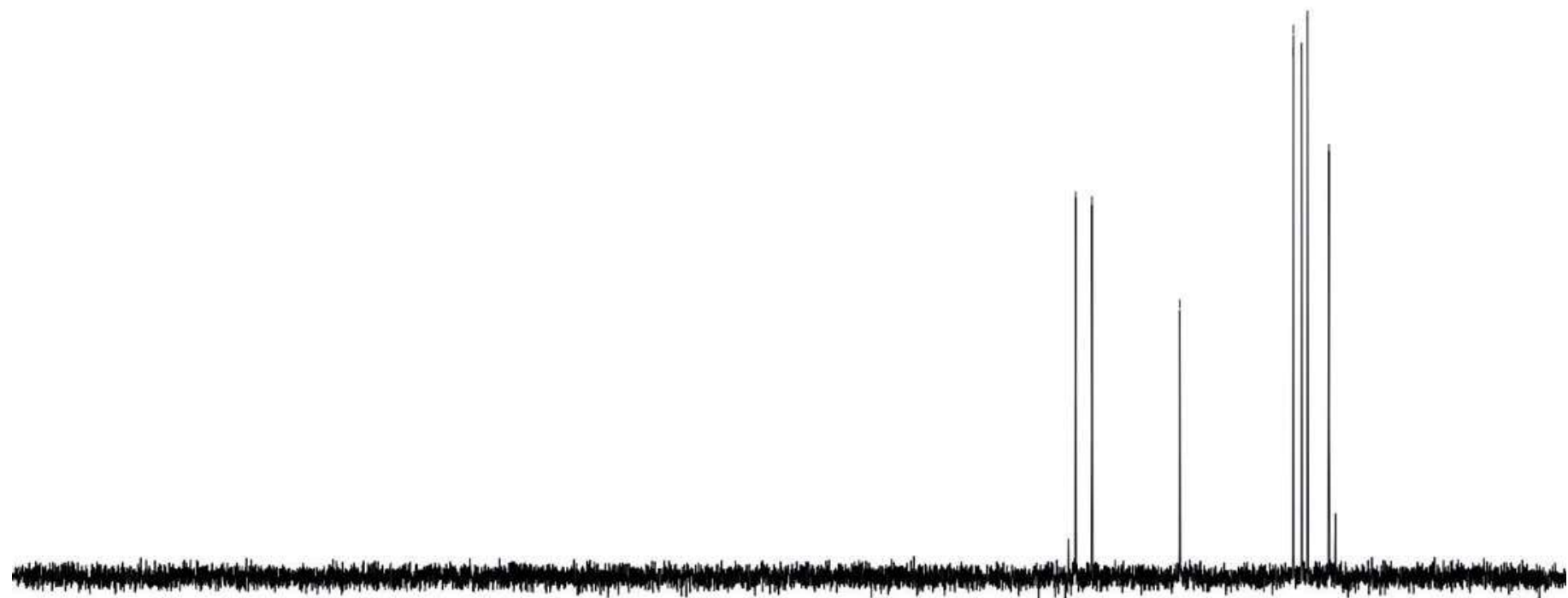

